\title{
Stellar Flares from the First TESS Data Release: Exploring a New Sample of M Dwarfs
}

\section{Günther, Maximilian N.; Zhan, Zhuchang; Seager, Sara; Rimmer, Paul B.; Ranjan, Sukrit; Stassun, Keivan G.; Oelkers, Ryan J.; Daylan, Tansu; Newton, Elisabeth; Kristiansen, Martti H.}

Total number of authors:

25

Published in:

Astrophysical Journal

Link to article, DOI:

$10.3847 / 1538-3881 / a b 5 d 3 a$

Publication date:

2020

Document Version

Publisher's PDF, also known as Version of record

Link back to DTU Orbit

Citation (APA):

Günther, M. N., Zhan, Z., Seager, S., Rimmer, P. B., Ranjan, S., Stassun, K. G., Oelkers, R. J., Daylan, T., Newton, E., Kristiansen, M. H., Olah, K., Gillen, E., Rappaport, S., Ricker, G. R., Vanderspek, R. K., Latham, D. W., Winn, J. N., Jenkins, J. M., Glidden, A., ... Ting, E. B. (2020). Stellar Flares from the First TESS Data Release: Exploring a New Sample of M Dwarfs. Astrophysical Journal, 159(2), [60]. https://doi.org/10.3847/1538-3881/ab5d3a

\section{General rights}

Copyright and moral rights for the publications made accessible in the public portal are retained by the authors and/or other copyright owners and it is a condition of accessing publications that users recognise and abide by the legal requirements associated with these rights.

- Users may download and print one copy of any publication from the public portal for the purpose of private study or research.

- You may not further distribute the material or use it for any profit-making activity or commercial gain

- You may freely distribute the URL identifying the publication in the public portal 


\title{
Stellar Flares from the First TESS Data Release: Exploring a New Sample of M Dwarfs
}

\author{
Maximilian N. Günther ${ }^{1,15}$ (1) , Zhuchang Zhan $^{2}$ (1), Sara Seager ${ }^{1,2}$, Paul B. Rimmer ${ }^{3,4,5,16}$ (1), Sukrit Ranjan ${ }^{2,17}$ (1), \\ Keivan G. $\operatorname{Stassun}^{6}$ (D), Ryan J. Oelkers ${ }^{6}$ (D), Tansu Daylan ${ }^{1,18}$ (D), Elisabeth Newton ${ }^{1,7,19}$ (D), Martti H. Kristiansen ${ }^{8,9}$, \\ Katalin Olah $^{10}$ (D), Edward Gillen ${ }^{4,20}$ (D), Saul Rappaport ${ }^{1}$ (D), George R. Ricker ${ }^{1}$, Roland K. Vanderspek ${ }^{1}$ (D), David W. Latham ${ }^{11}$ (D), \\ Joshua N. Winn ${ }^{12}$ (D) , Jon M. Jenkins ${ }^{13}$ (D), Ana Glidden ${ }^{1,2}$ (D), Michael Fausnaugh ${ }^{1}$ (D), Alan M. Levine ${ }^{1}$, Jason A. Dittmann ${ }^{2,21}$, \\ Samuel N. Quinn ${ }^{11}$ (D), Akshata Krishnamurthy ${ }^{14}$, and Eric B. Ting ${ }^{13}$ \\ ${ }^{1}$ Department of Physics, and Kavli Institute for Astrophysics and Space Research, Massachusetts Institute of Technology, Cambridge, MA 02139, USA \\ maxgue@mit.edu \\ ${ }^{2}$ Department of Earth, Atmospheric, and Planetary Sciences, Massachusetts Institute of Technology, 77 Massachusetts Avenue, Cambridge, MA 02139, USA \\ ${ }^{3}$ Department of Earth Sciences, University of Cambridge, Downing Street, Cambridge, CB2 3EQ, UK \\ 4 Astrophysics Group, Cavendish Laboratory, J.J. Thomson Avenue, Cambridge CB3 0HE, UK \\ ${ }^{5}$ MRC Laboratory of Molecular Biology, Francis Crick Avenue, Cambridge CB2 0QH, UK \\ ${ }^{6}$ Vanderbilt University, Department of Physics \& Astronomy, 6301 Stevenson Center Lane, Nashville, TN 37235, USA \\ ${ }^{7}$ Dartmouth College, Hanover, NH 03755, USA \\ ${ }^{8}$ DTU Space, National Space Institute, Technical University of Denmark, Elektrovej 327, DK-2800 Lyngby, Denmark \\ ${ }^{9}$ Brorfelde Observatory, Observator Gyldenkernes Vej 7, DK-4340 Tølløse, Denmark \\ ${ }^{10}$ Konkoly Observatory, CSFK, H-1121 Budapest, Konkoly Thege M. 'ut 15-17, Hungary \\ ${ }_{11}^{11}$ Center for Astrophysics, Harvard \& Smithsonian, 60 Garden Street, Cambridge, MA 02138, USA \\ ${ }^{12}$ Department of Astrophysical Sciences, Princeton University, 4 Ivy Lane, Princeton, NJ 08544, USA \\ ${ }^{13}$ NASA Ames Research Center, Moffett Field, CA 94035, USA \\ ${ }^{14}$ Department of Aeronautics and Astronautics, Massachusetts Institute of Technology, 77 Massachusetts Avenue, Cambridge, MA 02139, USA \\ Received 2019 January 2; revised 2019 October 21; accepted 2019 November 20; published 2020 January 20
}

\begin{abstract}
We perform a study of stellar flares for the 24,809 stars observed with 2 minute cadence during the first two months of the TESS mission. Flares may erode exoplanets' atmospheres and impact their habitability, but might also trigger the genesis of life around small stars. TESS provides a new sample of bright dwarf stars in our galactic neighborhood, collecting data for thousands of $\mathbf{M}$ dwarfs that might host habitable exoplanets. Here, we use an automated search for flares accompanied by visual inspection. Then, our public allesfitter code robustly selects the appropriate model for potentially complex flares via Bayesian evidence. We identify 1228 flaring stars, 673 of which are M dwarfs. Among 8695 flares in total, the largest superflare increased the stellar brightness by a factor of 16.1. Bolometric flare energies range from $10^{31.0}$ to $10^{36.9} \mathrm{erg}$, with a median of $10^{33.1} \mathrm{erg}$. Furthermore, we study the flare rate and energy as a function of stellar type and rotation period. We solidify past findings that fast rotating $\mathrm{M}$ dwarfs are the most likely to flare and that their flare amplitude is independent of the rotation period. Finally, we link our results to criteria for prebiotic chemistry, atmospheric loss through coronal mass ejections, and ozone sterilization. Four of our flaring $\mathrm{M}$ dwarfs host exoplanet candidates alerted on by TESS, for which we discuss how these effects can impact life. With upcoming TESS data releases, our flare analysis can be expanded to almost all bright small stars, aiding in defining criteria for exoplanet habitability.
\end{abstract}

Unified Astronomy Thesaurus concepts: Optical flares (1166); Exoplanets (498); Habitable planets (695); Red dwarf flare stars (1367); Stellar flares (1603); Stellar activity (1580); Habitable zone (696); Extrasolar rocky planets (511); Astrobiology (74); Pre-biotic astrochemistry (2079); Exoplanet atmospheres (487)

Supporting material: machine-readable tables

\section{Introduction}

The most extreme solar flare ever recorded, the "Carrington event," hit Earth in 1859 (Carrington 1859; Hodgson 1859). It released a flare energy of $10^{32} \mathrm{erg}$ and came accompanied by a coronal mass ejection (CME) which interacted with Earth's magnetic field and led to destructive consequences. Stellar flares like the Carrington event are explosive magnetic reconnection events in a star's magnetosphere, releasing bursts of isotropic radiation (see, e.g., Benz \& Güdel 2010; Shibata et al. 2016;

\footnotetext{
15 Juan Carlos Torres Fellow.

${ }^{16}$ SCOL Senior Fellow.

17 SCOL Postdoctoral Fellow.

18 Kavli Fellow.

19 NSF Astronomy and Astrophysics Postdoctoral Fellow.

20 Winton Fellow.

2151 Pegasi b Postdoctoral Fellow.
}

Doyle et al. 2018). Over short timescales of minutes to a few hours, they emit energy ranging from $10^{23}$ erg (nanoflares; e.g., Parnell \& Jupp 2000) to $10^{33}-10^{38}$ erg (superflares; e.g., Shibayama et al. 2013). Most of the emission is in the X-ray spectrum, but in extreme cases (like the Carrington event) flares are also visible in white light. Large flares can thus be detected with optical photometric surveys such as those dedicated for exoplanet detection. CMEs, on the other hand, are clouds of charged particles that get ejected into space with a constrained direction. Large flares are often accompanied by CMEs, but both events can also appear independently.

Flares and CMEs on stars hosting exoplanets can be even stronger and more frequent than those on the Sun, and can play a major role in planetary evolution and habitability. Flares may contribute to atmospheric erosion, destroy ozone layers on oxic planets, and act as stressors for surface life (e.g., Segura et al. 2003, 2010; Lammer et al. 2007; Scalo et al. 2007; Seager 2013; 
Atri 2017; Lingam \& Loeb 2017; O’Malley-James \& Kaltenegger 2018; Tilley et al. 2019). In the most extreme scenario, intense flare activity could render the immediate planet surface uninhabitable, though life could survive in the ocean, in rocks, or under shallow layers of soil or dust (Diaz \& SchulzeMakuch 2006; Kiang et al. 2007; Bryce et al. 2015; Estrela \& Valio 2018; Tilley et al. 2019). More optimistically, flares may also power prebiotic chemistry, produce surface biosignatures, or serve as a source for otherwise scarce visible-light photosynthesis on planets orbiting M dwarfs (Björn et al. 2015; Airapetian et al. 2016; Mullan \& Bais 2018). Indeed, recent work suggests that flares might be the only means for delivering the UV photons which have been proposed to be required to initiate life on exoplanets around M-dwarf stars (Ranjan et al. 2017; Rimmer et al. 2018). Finally, flares can alter the chemistry of planetary atmospheres, meaning their impact must be considered when interpreting atmospheric signals from a planetary atmosphere in search of biosignatures and geosignatures (Grenfell et al. 2012; Venot et al. 2016; Airapetian et al. 2017). In sum, it is critical to constrain the flare properties of exoplanet host stars to understand the evolution and habitability of their planets and to robustly characterize their atmospheres.

$\mathrm{M}$ dwarfs are of primary interest in the search for habitable exoplanets for several reasons. First, they constitute a large fraction $(\sim 70 \%)$ of the stellar population (Dole 1964; Henry et al. 1994; Reid et al. 2004; Covey et al. 2008). Second, their small radii and low temperatures enable the detection and atmospheric characterization of habitable planets on short orbits (Kaltenegger \& Traub 2009). For example, we know three exo-Earths in the "liquid-water habitable zone",2 of the red dwarf TRAPPIST-1 (Gillon et al. 2017), one around LHS 1140b (Dittmann et al. 2017), and one around Proxima Centauri (Anglada-Escudé et al. 2016). Nevertheless, other factors affecting the habitability of these systems remain largely unknown. More information on the frequency and energy of the host stars' flares can help better characterize the habitability of these planets.

Seminal work on flares from selected stars includes the study by Lacy et al. (1976) and was later expanded with surveys like the Sloan Digital Sky Survey (York et al. 2000; Kowalski et al. 2009). The largest number of flare discoveries to date come from distant stars observed by the Kepler mission (Borucki et al. 2010). Extensive catalogs of stellar flares from Kepler were provided by, e.g., Walkowicz et al. (2011), Hawley et al. (2014), Davenport (2016), and Van Doorsselaere et al. (2017). The adjusted observing strategy of $K 2$ allowed the study of bright M dwarfs in more detail (Stelzer et al. 2016). Groundbased wide-field surveys like the Next Generation Transit Survey (Wheatley et al. 2018), EVRYSCOPE (Law et al. 2015), and others have also contributed to flare catalogs for bright nearby objects (e.g., Howard et al. 2018; Jackman et al. 2018, 2019). Dedicated M-dwarf surveys like MEarth (Nutzman \& Charbonneau 2008) provide additional possibilities for flare studies for the smallest stars. For example, Mondrik et al. (2019) reported flares on 32 mid-to-late $\mathbf{M}$ dwarfs. Complementary, detailed spectroscopic monitoring campaigns of selected $\mathrm{M}$ dwarfs give insight into their flares over multiple wavebands

\footnotetext{
22 Note that definitions of the liquid-water habitable zone differ and depend on the planet mass, atmospheric composition, formation and migration history, tidal locking of the orbits, space weather, and other factors (see e.g., Huang 1959; Kasting et al. 1993, 2014; Pierrehumbert \& Gaidos 2011; Kopparapu et al. 2013, 2014; Cullum et al. 2014; Ramirez \& Kaltenegger 2014; Cullum \& Stevens 2016).
}

(e.g., MUSCLES; France et al. 2016). However, the number of bright $\mathrm{M}$ dwarfs observed for high-precision and high-cadence flare studies is still limited, and the limited precision of groundbased photometric observations only allows for the detection of the most energetic flares.

The TESS mission (Ricker et al. 2014), launched in 2018 April, provides the opportunity to study flares on early to late M dwarfs. TESS is specifically designed to observe bright, small stars in the solar neighborhood. It will photometrically monitor tens of thousands of $\mathrm{M}$ dwarfs, which are bright enough to allow the study of flares with a high signal-to-noise ratio $(\mathrm{S} / \mathrm{N})$.

Here, we present findings derived from the first two months of TESS data (i.e., sectors 1 and 2). Section 2 describes the TESS observations. Section 3 outlines our methodology for finding flare candidates, vet against false alarms, and model flare data to estimate the flare energy. The results are presented in Section 4, and a discussion and outlook are provided in Sections 5 and 6.

\section{Observations}

The primary goal of TESS is to search for transiting Earthsized planets around nearby and bright stars. Its four $10 \mathrm{~cm}$ optical cameras simultaneously observe a total field of $24^{\circ} \times 96^{\circ}$. In its two-year primary mission, TESS will measure lightcurves of over 200,000 preselected stars with a 2 minute cadence and of millions of stars with a 30 minute cadence.

This study is based on the short (2 minute) cadence data collected by TESS in sectors 1 and 2, which were made publicly available with the first data release in 2018 December. These data contain 24,809 unique targets: 8815 in sector 1 only, 8920 in sector 2 only, and 7074 in both sectors.

The 2 minute cadence data were extracted using the Science Processing Operations Center (SPOC) pipeline, a descendant of the Kepler mission pipeline (Jenkins 2002, 2017; Jenkins et al. 2010, 2016; Smith et al. 2012; Stumpe et al. 2014). The SPOC pipeline is operated at the NASA Ames Research Center.

\subsection{Searching for Flares}

To search for potential flaring stars, we analyze the 24,809 targets with TESS lightcurves from sectors 1 and 2. We start from the presearch data conditioned simple aperture (PDCSAP) lightcurves, which are detrended for instrument systematics. Additionally, we detrend each orbit using a spline fit to remove any remaining long-term variations in the lightcurves. We then remove strong periodicity from the lightcurves, which is generally caused by stellar variability or rotation. For this, we compute a Lomb-Scargle periodogram and remove a periodic signal using a sine wave fit if two criteria are fulfilled: first, the signal must have a false-alarm probability below 0.01 ; second, the standard deviation of the residuals must decrease. We repeat this process at maximum three times.

Next, we compute a running median using a global and a local view. For the global view, the local median flux and standard deviation, $\sigma$, is calculated using a 1024 data point bin (i.e., 1.4 days). This is meant to catch the largest and longest flares. For the local view, we use a 128 data bin (i.e., $4.3 \mathrm{hr}$ ) to catch smaller and shorter flares. We first run the global view iteratively and mask out all $3 \sigma$ outliers until no more are 

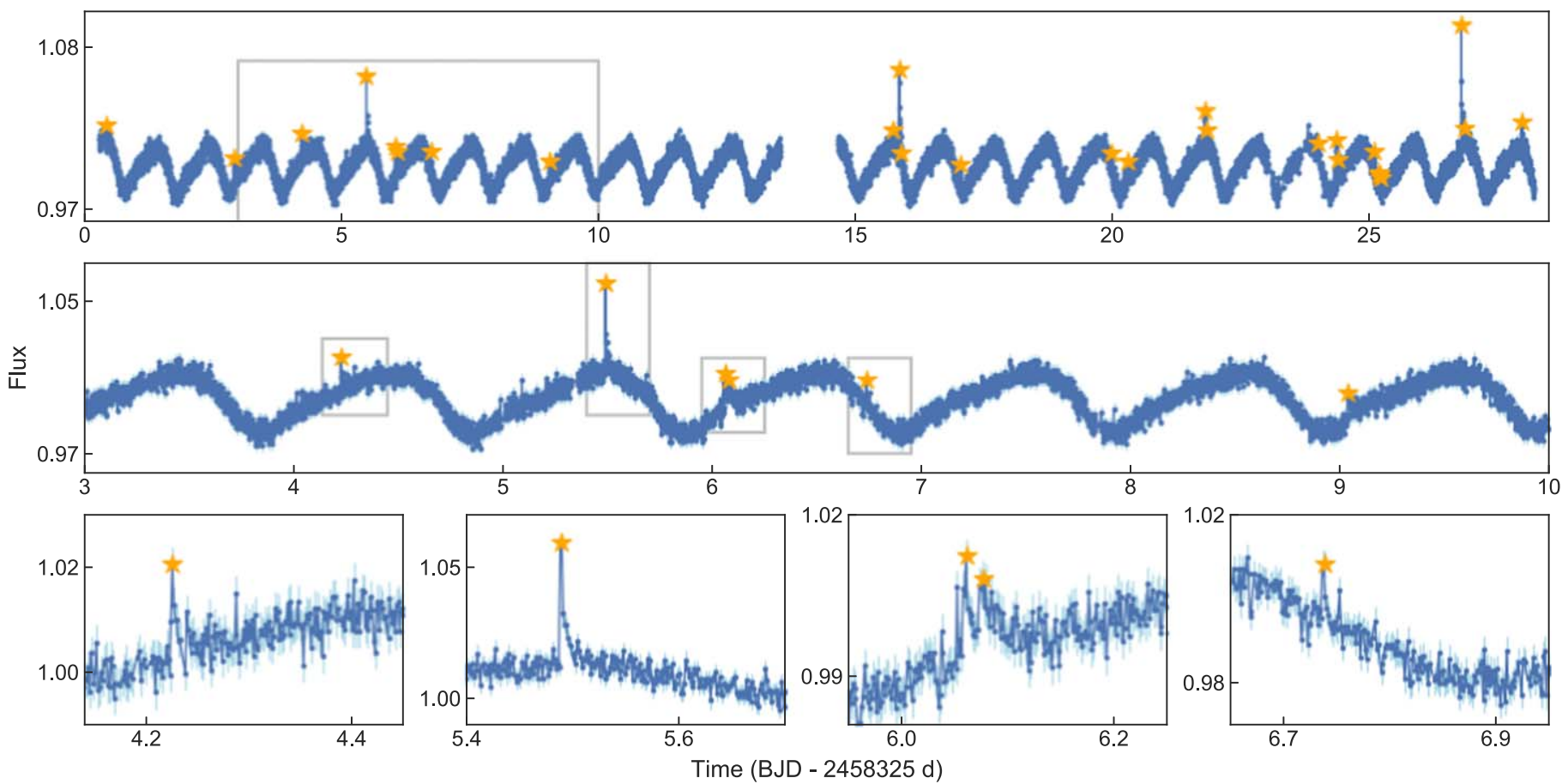

Figure 1. Demonstration of the detection pipeline on the example of the lightcurve of TIC 25118964. The $x$-axes show the time in Barycentric Julian Days (BJD) and $y$-axes show the normalized TESS PDC-SAP flux. Orange star symbols highlight the detected flare candidate peaks. The lower panels show zoomed views onto the regions marked with gray boxes. The flare candidate peaks are detected despite having small signal-to-noise and the presence of stellar rotation modulation.

detected. Afterwards, we run the local view with the same criterion and collect a list of all identified outliers.

We identify outliers as flare candidates if at least six minutes of flux data lie above a $3 \sigma$ threshold. These criteria are empirically selected to separate noise and actual flaring objects. Each target can (and often does) have multiple "flare candidate peaks." Figure 1 illustrates the methodology using TIC 25118964 as an example. The flare candidate peaks are detected despite having small $\mathrm{S} / \mathrm{N}$ and the presence of strong stellar rotation modulation. Finally, we visually inspect all candidate peaks to remove false positives (e.g., asteroids) and false alarms due to noise features.

\subsection{Defining Outburst Epochs}

Flares are often not isolated events. When the star is active, multiple consecutive flares may occur within a short time period. We denote this collection of flares as an "outburst." The effects of multiple flares during an outburst can overlap, resulting in compound features in the observed lightcurve.

We split each lightcurve into sections, one for each outburst epoch. To define which flares are part of which outburst epoch, we iterate through all flare events sorted in time. For a given flare, if there is no other candidate peak one hour before or three hours after the event, the outburst is labeled as containing only this single flare. When there are other flares following, the outburst epoch gets expanded accordingly. As a result, outburst epochs span from $1 \mathrm{hr}$ before their earliest flare peak to $3 \mathrm{hr}$ after their latest flare peak.

\subsection{Completeness of the Flare-detection Pipeline}

We evaluate the completeness of our flare-detection pipeline using two separate injection-recovery tests. For each sector, we randomly select lightcurves of $200 \mathrm{~F}, \mathrm{G}$, and K dwarfs; 200 early M dwarfs; and 200 late M dwarfs. In the first test sample (individual flares), we inject 10 flares into each lightcurve at random times, with amplitudes randomly drawn from a lognormal distribution between 0.01 and 1 in relative flux, and FWHMs randomly drawn from a uniform distribution between 2 minutes and $2 \mathrm{hr}$. In the second test sample (outbursts), we choose the same criteria, but force all flares to occur subsequently, mimicking extreme outburst regions. For this, we draw the first flare peak time randomly and all subsequent peak times from a uniform distribution between 10 and 30 minutes after each other. While this is a much more extreme outburst than ever occurs in reality, it serves to test the robustness of our pipeline.

We then run our flare-detection pipeline (Section 3.1) on all injected lightcurves and record which flares are recovered. In the individual flares sample, we consider a flare recovered if a detection is registered at less than 10 minute difference from the true peak time. In the outbursts sample, we consider an outburst recovered if any injected flare is recovered.

We find a clear lower limit for the flare amplitude, below which flares cannot be recovered (Figure 2). The recovery rate for F, G, and $\mathrm{K}$ dwarfs allows the detection of the smallest injected flares, with amplitudes at the millimagnitude level. For early M dwarfs, we find the recovery rates decrease for flares with amplitudes of less than a few percent. Flares on late $\mathrm{M}$ dwarfs must generally be at least a few percent to be detectable. This dependency on stellar types is a direct consequence of the stars' brightness, as for fainter stars (such as typical late $\mathrm{M}$ dwarfs) the photometric scatter (white noise) in the lightcurve increases, decreasing the $\mathrm{S} / \mathrm{N}$ of any flares. The detection efficiency shows only a slight dependency on the injected flare FWHM, increasing slightly for longer flares. Finally, as intrinsic stellar variability and rotation modulation are 

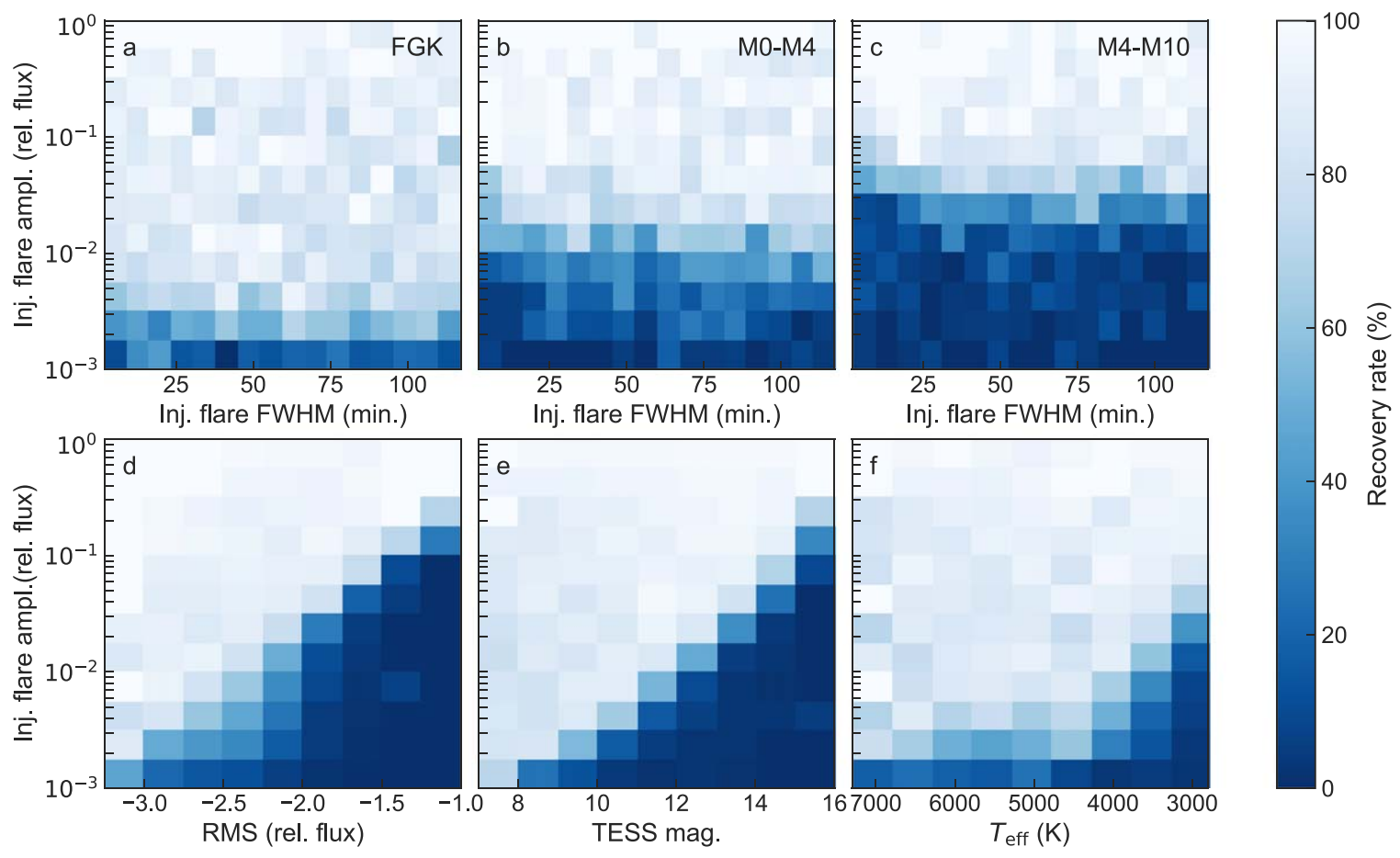

Figure 2. Recovery rates of flares from our injection tests. Upper panels show the recovery rate as a function of the injected flares' amplitudes and FWHM for (a) F, G, and K dwarfs, (b) early M dwarfs, and (c) mid/late M dwarfs. Lower panels show the recovery rate as a function of the (d) residual root mean squared (rms) error after detrending, (e) TESS magnitude, and (f) stellar effective temperature, all averaged over all stellar types. The different detection efficiency between stellar types is a direct consequence of their magnitudes, as the photometric scatter in the lightcurve increases for fainter stars.

detrended by our flare-detection pipeline, the minimum flare amplitude seems not to be affected.

\section{4. allesfitter}

allesfitter (Günther \& Daylan 2019, and M. N. Günther \& T. Daylan 2019, in preparation) is a publicly available, userfriendly software package for modeling photometric and RV data. It is based on a generative model that can accommodate multiple exoplanets, multistar systems, starspots, and stellar flares. For this, it constructs an inference framework that unites the versatile packages ellc (lightcurve and RV models; Maxted 2016), aflare (flare model; Davenport et al. 2014), dynesty (static and dynamic nested sampling; Speagle 2019), emcee (MCMC sampling; Foreman-Mackey et al. 2013), and celerite (GP models; Foreman-Mackey et al. 2017). allesfitter is publicly available at https:/github.com/ MNGuenther/allesfitter and is archived at Zenodo (Günther et al. 2019); feedback and contributions are welcome.

\subsection{Modeling Flares}

Using allesfitter, we perform nested sampling to infer flare models with up to $0,1, \ldots, N+2$ flares for each outburst epoch, where $N$ is the number of flare candidates. We sequentially add extra flares to ensure overlapping flares are distinguished even if they were missed by the detection pipeline and visual inspection. We start with fitting models of "only noise" and one flare. If the model with one flare passes, we test a model with two flares, and so on, up to a maximum of $N+2$.

The model selection is performed using two complementary criteria, which must be both fulfilled. First, adding an extra flare must increase the logarithm of the Bayesian evidence, $\log Z$, by at least 5 . Given a null model $M_{0}$, the alternative (more complex) model $M_{1}$ is only selected if there is sufficient relative Bayesian evidence for it, as quantified by Kass \& Raftery (1995). Hence, we define

$$
\begin{gathered}
\Delta \log Z:=\log Z_{M_{1}}-\log Z_{M_{0}}, \\
\sigma(\Delta \log Z):=\sqrt{\left[\sigma\left(\log Z_{M_{1}}\right)\right]^{2}-\left[\sigma\left(\log Z_{M_{0}}\right)\right]^{2}}
\end{gathered}
$$

and demand

$$
\begin{gathered}
\Delta \log Z>5, \\
\Delta \log Z>\sigma(\Delta \log Z) .
\end{gathered}
$$

Second, the extra flare must have an $\mathrm{S} / \mathrm{N}$ larger than 5 . For lightcurves with significant red noise, estimating the Bayesian evidence can be dominated by the volume of the prior and be biased if flares are misused to account for noise structures (i.e., the nested sampler could find numerous solutions for placing small flares). This risk can be mitigated by introducing an $\mathrm{S} / \mathrm{N}$ criterion.

We apply all fits to the detrended PDC-SAP flux. To additionally detrend any systematic noise or stellar variability features without affecting flares, we fit a Gaussian process with a Matern 3/2 kernel in parallel with all flare models. For each model, we first run two short Markov Chain Monte Carlo (MCMC) chains to explore the likelihood space and constrain the search space for the nested sampler. This also allows us to label the sequence of flares, mitigating the risk of label swapping during the nested sampling run. 


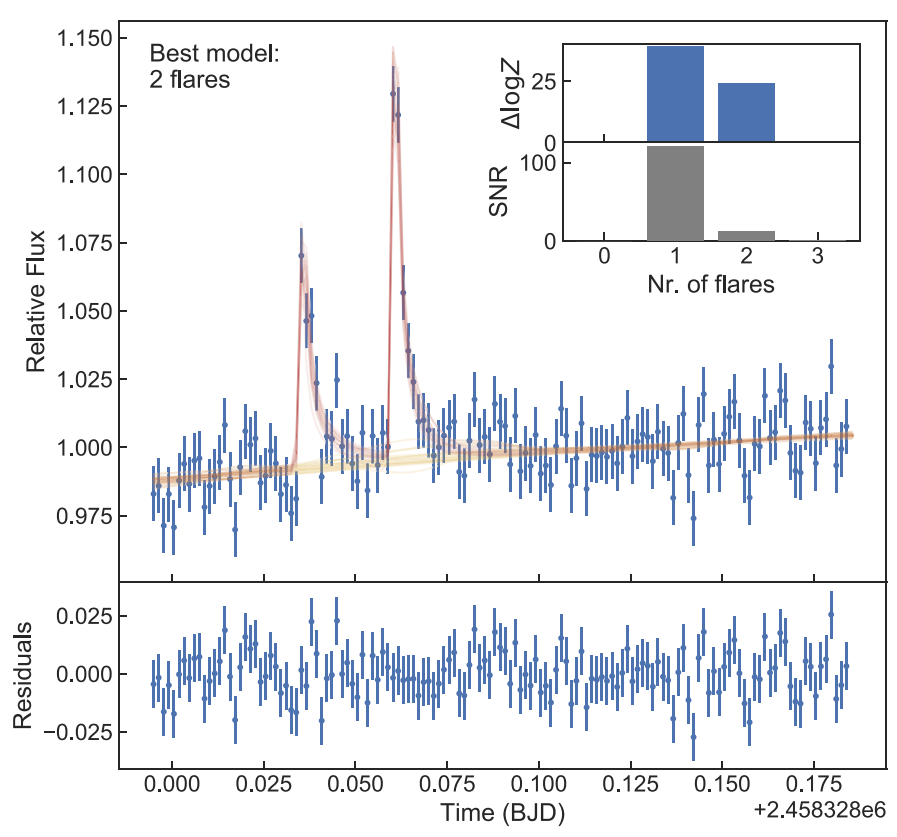

(a) TIC 139804406

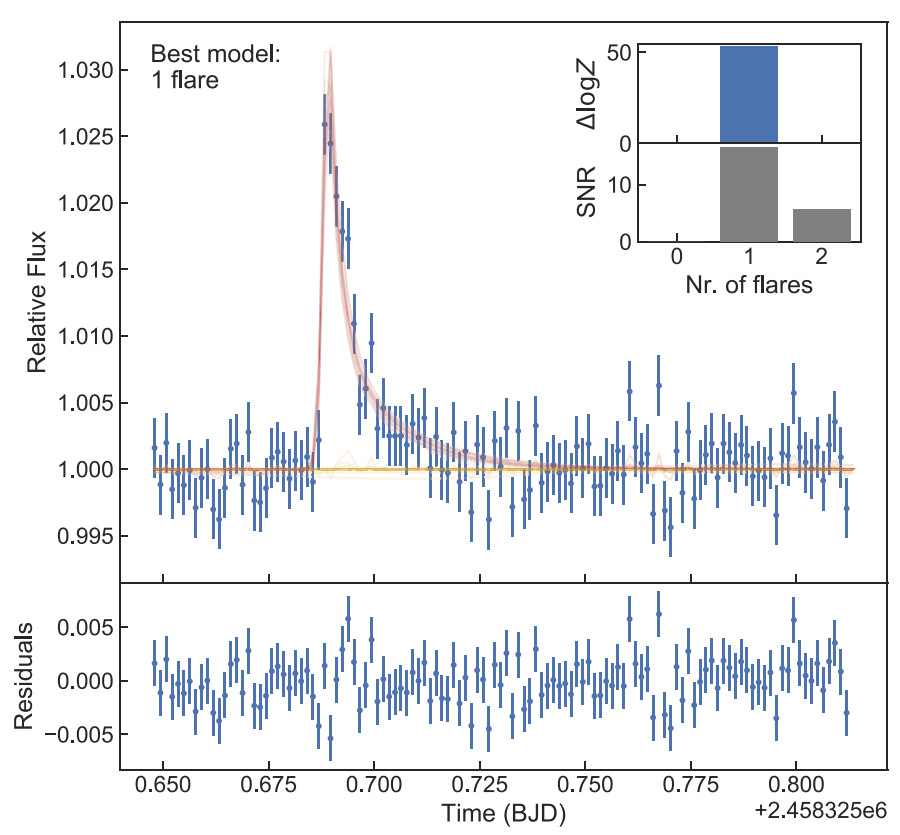

(c) TIC 144217628

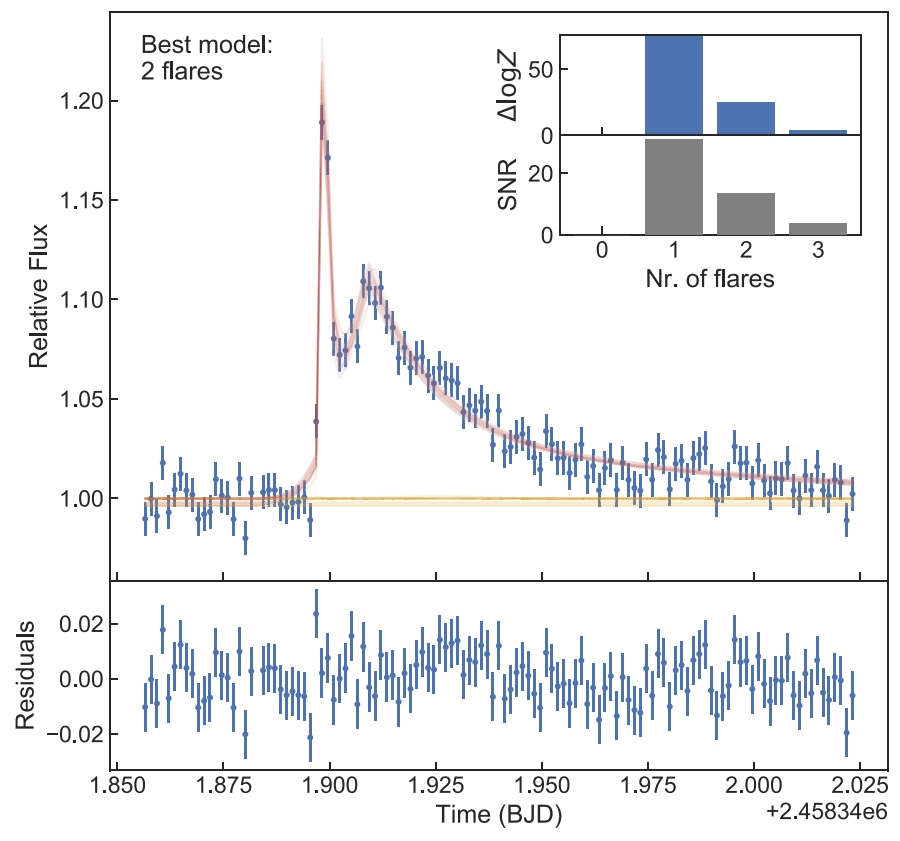

(b) TIC 129646813

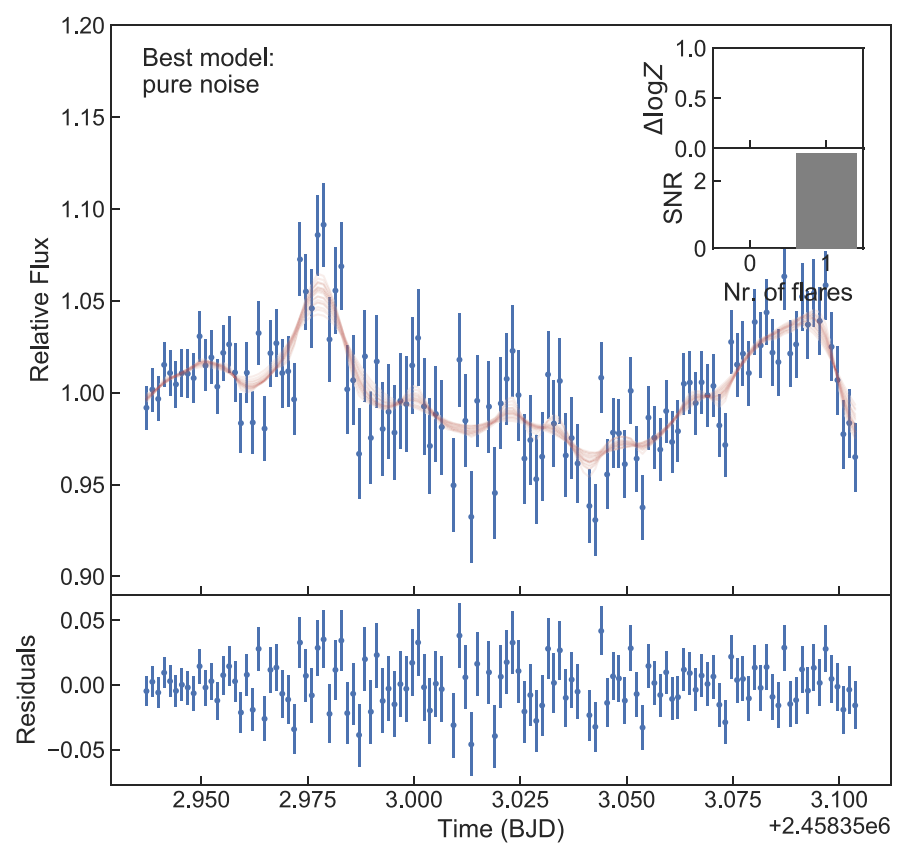

(d) TIC 152875048

Figure 3. Model fits of candidate outbursts of four example stars. In the main figures, the $x$-axis shows the time in Barycentric Julian Date (BJD) and the $y$-axis shows the TESS PDC-SAP flux. Red curves show 20 posterior samples generated by the best model, while orange curves show their corresponding baselines drawn from a Gaussian process with a Matern $3 / 2$ kernel. The curves for the less suitable models are not shown. The inlets show the gain in Bayesian evidence, $\Delta \log Z$, by adding an additional flare (upper inlay), and the $\mathrm{S} / \mathrm{N}$ of the additional flare (see Section 3.5). Initially, $N$ flare candidates were detected. Consequently, scenarios from 0 to at most $N+2$ flares are fitted as long as $\Delta \log Z>5$ and $\mathrm{S} / \mathrm{N}>5$ for an additional flare. This allows for a quantitative model comparison, confirming the suitable number of flares (see Section 3.5). (a) TIC 139804406: two candidate peaks were initially detected and then confirmed using our model fit; introducing a third flare does not lead to any gain in Bayesian evidence. (b) TIC 129646813: only one candidate peak was detected, but our model comparison confirmed two flares; adding a third flare is not favored. (c) TIC 144217628: the Bayesian evidence rejects the scenario where the smaller peak is a flare; instead, it favors the peak being within the limits of a noise feature. (d) TIC 152875048: one candidate peak was detected, but the fit favors the pure noise model and rejects a flare scenario.

Figure 3 illustrates our methodology on four example targets. For TIC 139804406, we detected two flare candidates in the shown outburst epoch. Comparing all plausible models, we can confirm this scenario. For TIC 129646813, the candidate list initially consisted of a single flare. However, our model fit and comparison show that there are, in fact, two flares. For TIC 144217628, there is no evidence that the short feature toward the end of the outburst is a flare; it is best explained as a noise feature. Finally, for TIC 52875048, the candidate peak was identified as a noise feature.

Note that the Bayesian evidence helps determine the degree to which a model is supported by the data and does not simply 
Table 1

Catalog of All Individual Flares Found in TESS Sectors 1 and 2

\begin{tabular}{|c|c|c|c|c|c|c|c|c|}
\hline TIC ID & Sector & Outburst & Flare & $\begin{array}{c}t_{\text {peak }} \\
\text { (BJD) }\end{array}$ & $\begin{array}{c}\text { Amp. } \\
\text { (rel. flux) }\end{array}$ & $\begin{array}{c}\text { FWHM } \\
\text { (day) }\end{array}$ & $\begin{array}{l}E_{\text {bol }} \\
\text { (erg) }\end{array}$ & $\begin{array}{l}M_{\mathrm{CME}} \\
(\mathrm{g})\end{array}$ \\
\hline \multicolumn{9}{|c|}{$\cdots$} \\
\hline 167602025 & 2 & 2 & 1 & 2458373.524 & 0.008 & 0.006 & $8.15 \mathrm{e}+33$ & $3.42 \mathrm{e}+20$ \\
\hline 167695269 & 1 & 1 & 1 & 2458336.636 & 0.157 & 0.004 & $2.18 \mathrm{e}+33$ & $1.71 \mathrm{e}+20$ \\
\hline 167695269 & 1 & 2 & 1 & 2458343.026 & 0.512 & 0.002 & $4.25 \mathrm{e}+33$ & $2.74 \mathrm{e}+20$ \\
\hline 167696018 & 1 & 1 & 1 & 2458325.491 & 0.010 & 0.010 & $5.43 e+33$ & $3.23 e+20$ \\
\hline
\end{tabular}

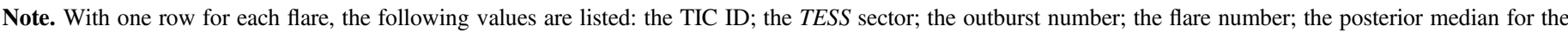

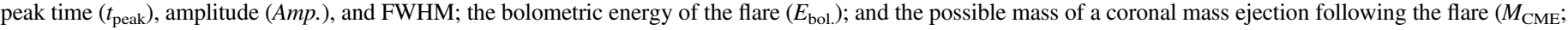

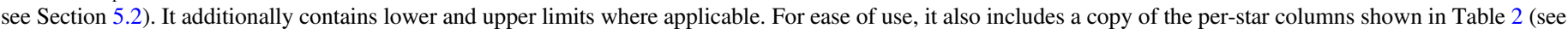
below).

(This table is available in its entirety in machine-readable form.)

indicate its likelihood. For example, for TIC 129646813 (Figure 3(b)), we have $\Delta \log Z_{1 \rightarrow 2 \text { flares }}=25$. This is not to be confused with a statement such as "two flares are 25 times more likely than one flare." Instead, it implies that there is very strong evidence for the model with two flares.

\subsection{Vetting for Asteroids and Blends}

We inspected a sample of around 100 strongly flaring stars for misclassified flares caused by solar system objects (SSO). This effect was discussed by Pál et al. (2018) for the TESS mission. Additional catalog inputs within the same sector as the high amplitude peaks were included in our sample, making it a total of circa 600 flare events. We used SkyBot (Berthier et al. 2006, 2016) to search for known SSOs crossing the target stars at the time of flare peaks. In only a few cases did we find SSOs within a range of $100^{\prime \prime}$. In these instances, we carried out additional searches by slightly changing the input time, in order to see whether the same objects would be redetected. This was not the case in any of the computations, and we therefore discard these SSOs as potential contaminants. In a single occurrence, associated with TIC 160074646 at 2458379.648 $\mathrm{BJD}_{\mathrm{TDB}}$, we notice the nearby presence $\left(21\right.$ !' $\left.^{\prime}\right)$ of the outer main belt SSO 2010 KM11 (519036). However, the lightcurve profile of this event matches that of a flare profile with a fast rise and an exponential decay. Additionally, we created a series of pixel-level animations which we then visually inspected, similar to Szabó et al. (2015), to prevent the appearance of currently unknown SSOs in the sample. No SSO crossings were detected, including $2010 \mathrm{KM} 11$, most likely due to its visual magnitude of 20.7 .

In the process, we also checked for contamination by blended flaring stars. A low number of potential duplicate stars is marked in the column duplicates in Tables 1 and 2. For these, it was not possible to determine the true origin of the flaring, as the stars fall within the same pixel and potentially are part of a binary system. We also noted a single instance for TIC 229147922 at $2458363.726719 \mathrm{BJD}_{\mathrm{TDB}}$, in which one of the flares originated from a neighboring star.

\subsection{Measuring the Flare Energy}

We calculate the flare energy from the stellar luminosity and the best-fitting flare profile, following Shibayama et al. (2013).
The quiescent stellar luminosity $L_{\star}$ is retrieved from TESS Input Catalog (TIC) version 8 if available. If the TIC lists only the effective temperature $T_{\text {eff }}$ and stellar radius $R_{\star}$, we compute $L_{\star}$ from these parameters. If only the $T_{\text {eff }}$ is available, we estimate $R_{\star}$ using the updated values from Pecaut \& Mamajek $(2013)^{23}$ to then calculate $L_{\star}$.

We model the flare luminosity as blackbody radiation with an effective temperature of $9000 \pm 500 \mathrm{~K}$, as a conservative lower limit consistent with previous studies (e.g., Hawley \& Pettersen 1991; Kowalski et al. 2013; Shibayama et al. 2013; Davenport 2016; Chang et al. 2018; Howard et al. 2018; Jackman et al. 2018, 2019). As discussed, e.g., by Shibayama et al. (2013), the luminosities of the star and the flare in the observing bandpass ( $L_{\star}^{\prime}$ and $L_{\text {flare }}^{\prime}$, respectively) are given as

$$
\begin{gathered}
L_{\star}^{\prime}=\pi R_{\star}^{2} \int R_{\lambda} B_{\lambda}\left(T_{\text {eff }}\right) d \lambda, \\
L_{\text {flare }}^{\prime}(t)=A_{\text {flare }}(t) \int R_{\lambda} B_{\lambda}\left(T_{\text {flare }}\right) d \lambda .
\end{gathered}
$$

Here, $R_{\lambda}$ is the TESS response function, which is the product of the filter transmission and the detector quantum efficiency. ${ }^{24}$ Note that the normalization of $R_{\lambda}$ is irrelevant here, as it cancels out in the calculations below. $B_{\lambda}\left(T_{\text {eff }}\right)$ and $B_{\lambda}\left(T_{\text {flare }}\right)$ are the Planck functions evaluated for the star's effective temperature and the flare temperature. Last, $A_{\text {flare }}(t)$ is the area of the flare. Note that this assumes that the flare temperature is constant throughout.

Because the normalized lightcurve gives us the relative flare amplitude $(\Delta F / F)(t)=L_{\text {flare }}^{\prime}(t) / L_{\star}^{\prime}$, we can solve these equations for $A_{\text {flare: }}$

$$
A_{\text {flare }}(t)=(\Delta F / F)(t) \pi R_{\star}^{2} \frac{\int R_{\lambda} B_{\lambda}\left(T_{\text {eff }}\right) d \lambda}{\int R_{\lambda} B_{\lambda}\left(T_{\text {flare }}\right) d \lambda} .
$$

This leads to the bolometric flare luminosity $L_{\text {flare: }}$ :

$$
L_{\text {flare }}=\sigma_{\mathrm{SB}} T_{\text {flare }}^{4} A_{\text {flare }} .
$$

\footnotetext{
23 http://www.pas.rochester.edu/ emamajek/EEM_dwarf_UBVIJHK_ colors_Teff.txt, online 2018 December 28.

24 https://heasarc.gsfc.nasa.gov/docs/tess/data/tess-response-function-v1.0. csv, online 2018 December 28.
} 
Table 2

Catalog of All Flaring Stars Found in TESS Sectors 1 and 2

\begin{tabular}{|c|c|c|c|c|c|c|c|c|c|c|c|c|c|c|c|c|c|}
\hline TIC ID & $N_{\text {sec. }}$ & $N_{\text {out. }}$ & $N_{\text {fla. }}$ & $\begin{array}{c}\text { Amp. } \\
\text { max. } \\
\text { (flux - 1) }\end{array}$ & $\begin{array}{l}\cdots \\
\cdots \\
\cdots\end{array}$ & $\begin{array}{c}M_{\mathrm{CME}} \\
\text { mean } \\
(\mathrm{g})\end{array}$ & $\begin{array}{c}\text { TESS } \\
\text { mag }\end{array}$ & $\begin{array}{l}T_{\text {eff }} \\
(\mathrm{K})\end{array}$ & $\begin{array}{c}R_{\star} \\
\left(R_{\odot}\right)\end{array}$ & $\begin{array}{c}\log g \\
\left(\log \mathrm{cm} \mathrm{s}^{-2}\right)\end{array}$ & $\begin{array}{c}P_{\text {rot }} \\
T E S S \\
\text { (days) }\end{array}$ & $\begin{array}{c}P_{\text {rot }} \\
\text { KELT } \\
\text { (days) }\end{array}$ & $\alpha_{\mathrm{FFD}}$ & $\beta_{\mathrm{FFD}}$ & $\begin{array}{l}\text { preb. } \\
\text { chem. }\end{array}$ & $\begin{array}{l}\text { ozone depl. } \\
\text { cons. }\end{array}$ & $\begin{array}{c}\text { ozone depl. } \\
\text { perm. }\end{array}$ \\
\hline 167602025 & 2 & 5 & 7 & 0.061 & $\ldots$ & $2.18 \mathrm{E}+21$ & 11.277 & 5341 & $\begin{array}{r}\ldots \\
0.944\end{array}$ & $\ldots$ & 2.477 & 0.719 & -0.677 & 22.236 & no & yes & no \\
\hline 167695269 & 1 & 3 & 3 & 0.512 & $\ldots$ & $2.74 \mathrm{E}+20$ & 13.001 & 3381 & 0.374 & 4.851 & $\ldots$ & $\ldots$ & $\ldots$ & $\ldots$ & no & no & no \\
\hline 167814740 & 2 & 8 & 11 & 0.589 & $\ldots$ & $6.62 \mathrm{E}+20$ & 13.243 & 3283 & 0.524 & 4.717 & 0.403 & $\ldots$ & -0.586 & 18.594 & no & no & no \\
\hline 102032397 & 1 & 11 & 13 & 0.093 & $\ldots$ & $1.68 \mathrm{E}+21$ & 10.593 & 5435 & 1.105 & 4.329 & 3.715 & 1.343 & -1.045 & 35.482 & no & yes & yes \\
\hline
\end{tabular}

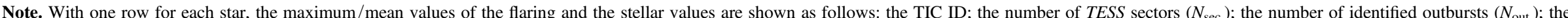

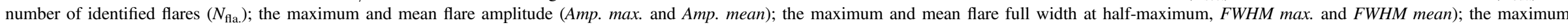

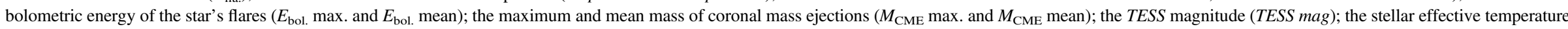

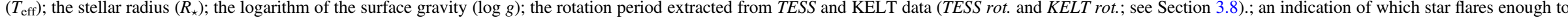

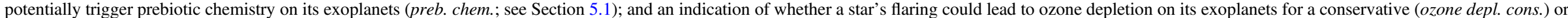

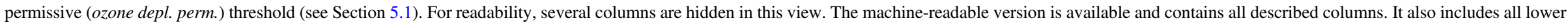
and upper limits values where applicable.

(This table is available in its entirety in machine-readable form 
Finally, we arrive at the expression for the bolometric energy of the flare, given as

$$
E_{\text {flare }}=\int L_{\text {flare }}(t) d t
$$

We estimate the error on the flare energy by propagating all uncertainties on the stellar properties from the TIC and the estimated uncertainty on the flare temperature.

\subsection{Identifying Stellar Rotation Periods}

The rate and energy of stellar flares depend on the surface magnetic activity of the star; thus, flares are thought to result from strong dynamo activity (e.g., Parker 1979). Accordingly, the flaring activity should depend on the effective temperature and rotation periods (elaborated in Section 4.3).

We derive the stellar rotation period of all flaring and nonflaring stars from the TESS lightcurves themselves and from a study conducted with the Kilodegree Extremely Little Telescope (KELT) by Oelkers et al. (2018). For TESS data, we measure the rotation period $\left(P_{\text {rot }}\right)$ using Fast Fourier Transform (FFT) computations (see Zhan et al. 2019 for details). We choose the FFT method due to its robustness and computational speed. With the data being uniformly sampled, there was no advantage in using Lomb-Scargle transforms (Lomb 1976; Scargle 1982) or other methods. We detect and analyze the FFT frequency peaks to find the primary rotation period. This is sensitive to the Nyquist limit at 4 minutes and a conservative upper limit of 5 days (to minimize false alarms due to momentum dumps and the 13 day spacecraft orbits). The reported periods and uncertainties are then determined using a Stellingwerf transform (Stellingwerf 1978). Finally, the raw lightcurve is phase-folded onto the rotation period and examined by eye, ensuring that the signals are not caused by instrumental systematics or astrophysical false positives.

The 28 day baseline per TESS sector does not favor the identification of longer rotation periods, which are common among M-dwarf stars. Studies measuring the rotation periods of nearby low-mass stars (Newton et al. 2016b, 2018) have found a population of fast rotators $\left(P_{\text {rot }}<10\right.$ days $)$ and a population of slow rotators $\left(P_{\text {rot }}>70\right.$ days), and a dearth of objects in between. Considering only their highest quality lightcurves, Newton et al. (2018) found that two-thirds of mid-to-late M dwarfs have rotation periods longer than 28 days, the length of one TESS sector, and half have periods longer than 90 days.

KELT has performed high-cadence (10-30 minutes), timeseries photometric observations for more than four million sources since 2007. KELT observations have surveyed more than $70 \%$ of the celestial sphere, reaching a limiting magnitude of about $V=13$, and with a baseline of nine years using KELT North and five years using KELT South. Oelkers et al. (2018) provide a catalog of 52,741 objects showing significant photometric fluctuations likely caused by stellar variability, as determined via the Welch-Stetson $J$ and $L$ statistics (Stetson 1996). Additionally, this catalog includes 62,229 objects identified with likely stellar rotation periods as determined by a Lomb-Scargle periodogram analysis (Lomb 1976; Scargle 1982). The detected variability ranges in rms amplitude from $3 \mathrm{mmag}$ to $2.3 \mathrm{mag}$, and the detected periods range from 0.1 days to over 2000 days. Oelkers et al. (2018) also provide variability upper limits for all other four million sources observed by KELT. These upper limits typically have $1 \sigma$ sensitivity on 30 minute timescales down to 5 mmag at $V=8$, and 43 mmag at $V=13$.

\section{Results \\ 4.1. TESS Catalog of Stellar Flares}

All detected, vetted, and modeled flare events are summarized in Table 1 per flare event, and in Table 2 per star. In Table 1, each star can be listed multiple times, with one row per flare. For each flare event, the columns show the following: the TIC ID; the TESS sector; the outburst number; the flare number; the posterior medians for the peak time $\left(t_{\text {peak }}\right)$, amplitude (Amp.) and FWHM; the bolometric energy of the flare $\left(E_{\mathrm{bol}}\right)$; and the possible mass of a CME following the flare $\left(M_{\mathrm{CME}}\right.$; see Section 5.2). The machine-readable version is available, and additionally contains lower and upper limits. For ease of use, it also includes a copy of the per-star columns shown in Table 2 (see below).

In Table 2, each star only has one row; columns show the maximum/mean values of the flaring and the stellar parameters as follows: the TIC ID; the number of TESS sectors $\left(N_{\text {sec. }}\right)$; the number of identified outbursts $\left(N_{\text {out }}\right)$; the number of identified flares $\left(N_{\text {fla. }}\right)$; the maximum and mean flare amplitude (Amp. max. and Amp. mean); the maximum and mean flare full width at half-maximum ( $F W H M$ max. and $F W H M$ mean); the maximum bolometric energy of the star's flares $\left(E_{\mathrm{bol}}\right.$ max. and $E_{\text {bol. }}$ mean); the maximum and mean mass of CMEs $\left(M_{C M E}\right.$ max. and $M_{\mathrm{CME}}$ mean); the TESS magnitude (TESS mag); the stellar effective temperature $\left(T_{\text {eff }}\right)$; the stellar radius $\left(R_{\star}\right)$; the logarithm of the surface gravity $(\log g)$; the rotation period extracted from TESS and KELT data (TESS rot. and KELT rot.; see Section 3.8); an indication of which star flares enough to potentially trigger prebiotic chemistry on its exoplanets (preb. chem.; see Section 5.1); and an indication of whether a star's flaring could lead to ozone depletion on its exoplanets for a conservative (ozone depl. cons.) or permissive (ozone depl. perm.) threshold (see Section 5.1); the TIC IDs of any potential blends (duplicates). For readability, several columns are hidden in the printed version of Table 2. A machine-readable version is available and contains all described columns, among all lower and upper limits values.

\subsection{TESS Explores a Large Sample of Flares on Bright Early and Late $M$ Dwarfs}

We identify 1228 flaring stars among a total of 24,809 targets with short-cadence TESS observations in sectors 1 and 2 (Figure 4). This includes 531 flaring early M dwarfs (M0-M4; 3905-3200 K) and 142 flaring late $M$ dwarfs (M4-M10; $3200-2285 \mathrm{~K})$. Figure 5 shows the subsample of $M$ dwarfs categorized by stellar type. Flares are most commonly detected on mid M dwarfs, which is partly influenced by the TESS target selection and $\mathrm{S} / \mathrm{N}$ constraints for the flare detection. $\mathrm{M}$ dwarfs of type M4-M6 show the highest fraction of flaring stars.

Mid to late $\mathrm{M}$ dwarfs are the most common flare stars, with more than $40 \%$ of these showing observable flares (Figure 4(B)). This occurrence rate is significantly lower for early $\mathrm{M}$ dwarfs, as only $\sim 10 \%$ have observable flares. Note that this might directly relate to the convection limit, where the full convection of $\mathrm{M}$ dwarfs is supposed to start around spectral type M4 (e.g., Stassun et al. 2011). Hotter stars of type K and G seem to rarely host flaring events large enough to be detected (only $\sim 5 \%$ of these stars in our sample). We find flares only on 


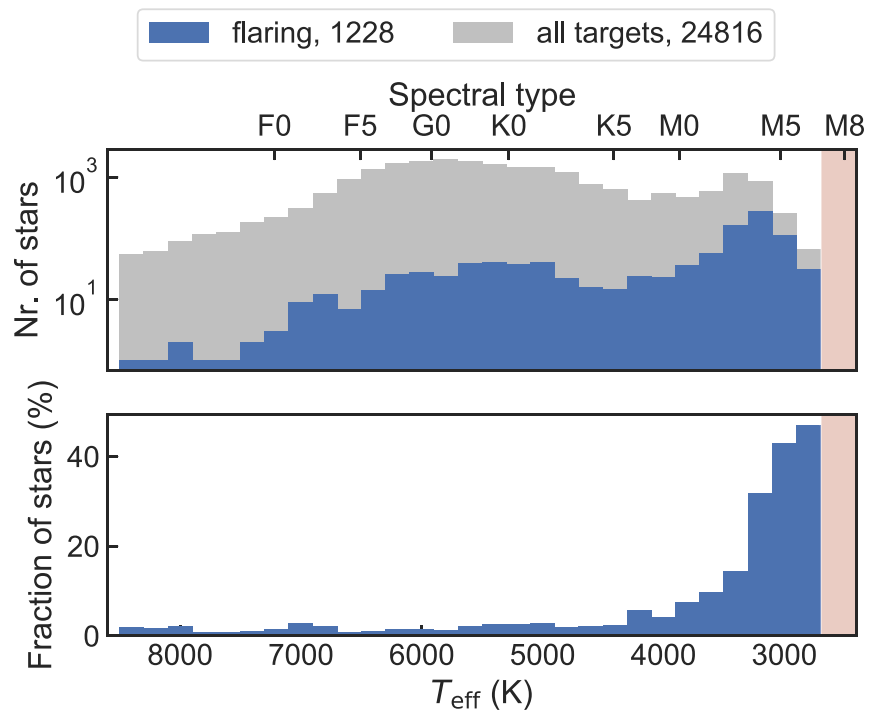

Figure 4. Histograms of the number (upper panel) and fraction (lower panel) of flaring stars (blue) compared with the total number of stars (gray) in the TESS short-cadence observations of sectors 1 and 2, shown as a function of the stellar effective temperature $T_{\text {eff }}$. The top axis indicates stellar types following the classification by Pecaut \& Mamajek (2013). M dwarfs dominate the sample of flaring stars, while $\mathrm{F}, \mathrm{G}$, and $\mathrm{K}$ stars rarely have detectable flares. We note that for later M dwarfs, the sample size is smaller (or zero), due to the TESS target selection. Additionally, the flare detection is limited by signal-to-noise constraints (see Section 3.3).

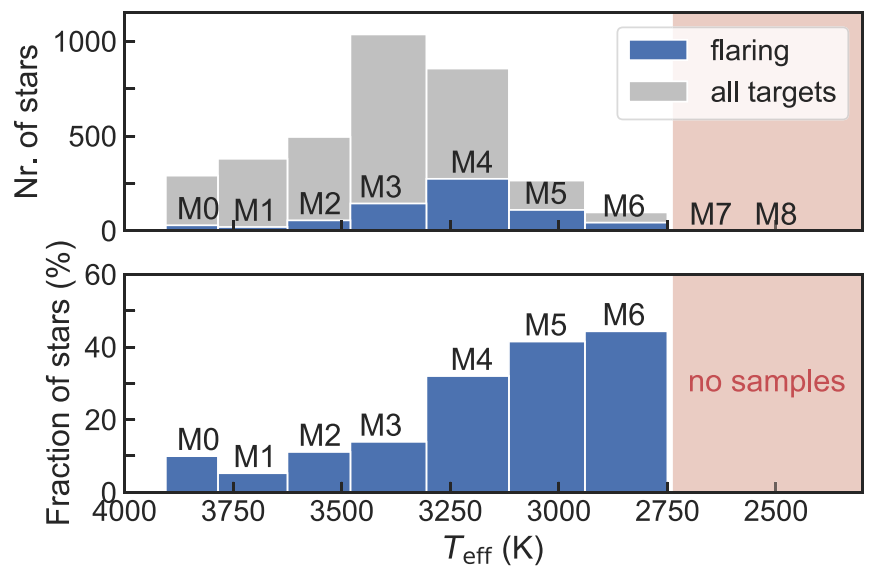

Figure 5. Variation of Figure 4 focused on M dwarfs, with bins matching the stellar types following the classification by Pecaut \& Mamajek (2013). Mid M dwarfs of type M4-M6 constitute both the highest number (upper panel) and highest fraction (lower panel) of flaring stars, with up to $30 \%$ of these having flares. Earlier M dwarfs seem to flare less, showing a significantly lower fraction of 5\%-10\%. Later $\mathrm{M}$ dwarfs were not observed in a large-enough sample size. We note that especially for M dwarfs later than M4 the sample size is lower due to the TESS target selection (favoring bright stars), and the flare detection is limited by signal-to-noise constraints (see Section 3.3).

$85 \mathrm{~F}, 114 \mathrm{G}$, and $184 \mathrm{~K}$ stars, all of which have low amplitudes. This confirms previous observations that $\mathrm{M}$ dwarfs flare more frequently and strongly than F, G, and $\mathrm{K}$ stars.

However, we note the interplay of two detection biases. Flares on $\mathrm{M}$ dwarfs have a strong white light contribution and a stronger contrast against their red spectra and low quiescent luminosity. This enhances $\mathrm{M}$ dwarfs' observable amplitudes in the TESS band, favoring the detection of less energetic flares. On the other hand, $\mathrm{M}$ dwarfs also have higher photometric noise, which in turn decreases the $\mathrm{S} / \mathrm{N}$ of any flares (see Section 3.3). For F, G, and $\mathrm{K}$ dwarfs, on the other hand, only

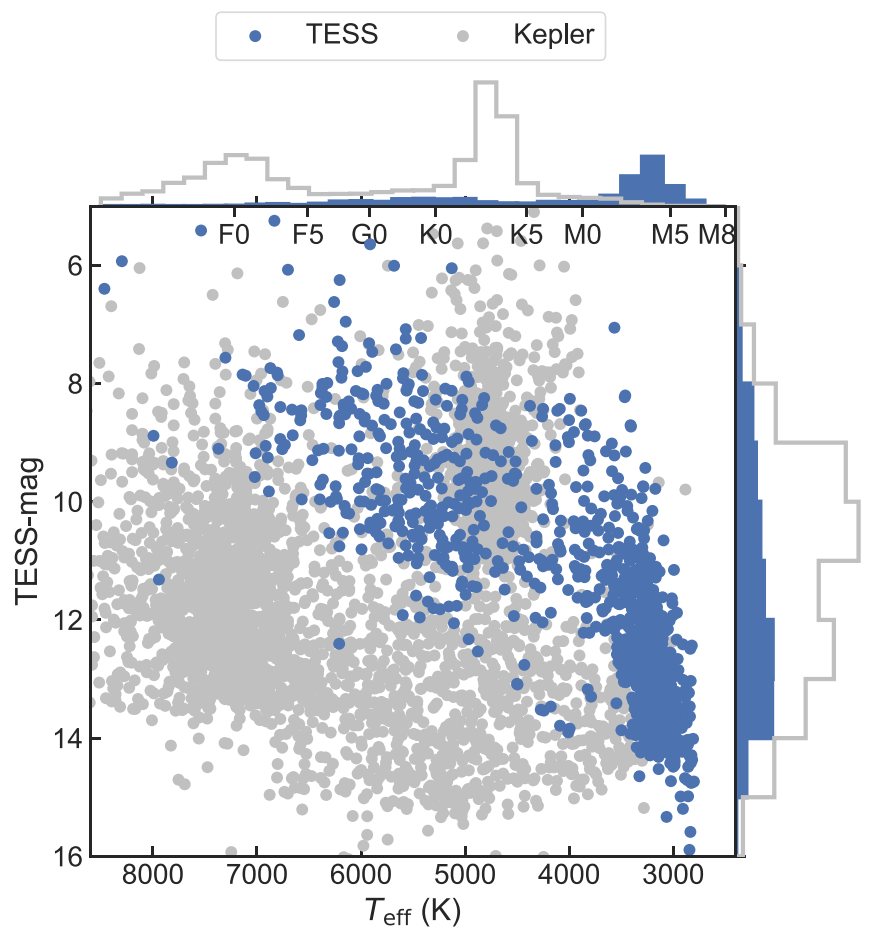

Figure 6. TESS explores bright early to late $M$ dwarfs, expanding the sample size for flare studies in this parameter space. Shown are the effective temperature $T_{\text {eff }}$ vs. TESS magnitude of flaring stars in the TESS sample (blue) and the Kepler flare catalog (gray; Davenport 2016). The top axis indicates stellar types following the classification by Pecaut \& Mamajek (2013). The first two sectors already include 531 early M dwarfs and 142 late M dwarfs. Out of these, 189 and 6 are brighter than 12th TESS magnitude. In addition, TESS detects small flares on $\mathrm{F}, \mathrm{G}$, and $\mathrm{K}$ dwarfs that are brighter than the average Kepler targets.

the most energetic flares cause high-enough amplitudes to be detected in contrast to their quiescent brightness in white light. Yet, favorable for these stars is their lower photometric noise in the TESS band.

TESS is designed to survey bright dwarf stars, expanding the number of M-dwarf flares detected in the solar neighborhood (Figure 6). Our sample includes 189 early M dwarfs and 6 late M dwarfs brighter than the 12th TESS magnitude. These stars can be prime targets for atmospheric characterization and radial velocity follow-up if they are found to host transiting planets. Flares can provide constraints on the existence of an atmosphere or habitability constraints (see Section 5).

With 24 more sectors to be observed in the next two years, and flare detection in the full-frame images, the expected TESS yield of flaring $\mathrm{M}$ dwarfs is on the order of $10^{4}$. In contrast, the Kepler mission focused on a different sample, namely F, G, and $\mathrm{K}$ stars (see Figure 6). The Kepler flare catalog (Davenport 2016) collects a total of 4041 objects. Of these, 4036 can be cross-matched with the TIC. The cross-matched list contains 110 flaring early $M$ dwarfs and 4 flaring late $M$ dwarfs observed with Kepler. Additional studies with Kepler (e.g., Van Doorsselaere et al. 2017), K2 (Stelzer et al. 2016), MEarth (Mondrik et al. 2019), and others can provided an expanded view into the M-dwarf regime, yet still for limited sample sizes. Figure 6 highlights how TESS enables exploring this parameter space for flare studies in an unprecedented manner. Moreover, TESS also detects small flares on $\mathrm{F}, \mathrm{G}$, and $\mathrm{K}$ dwarfs brighter than the 12th TESS magnitude, filling in another parameter space which was less explored by the Kepler mission. 


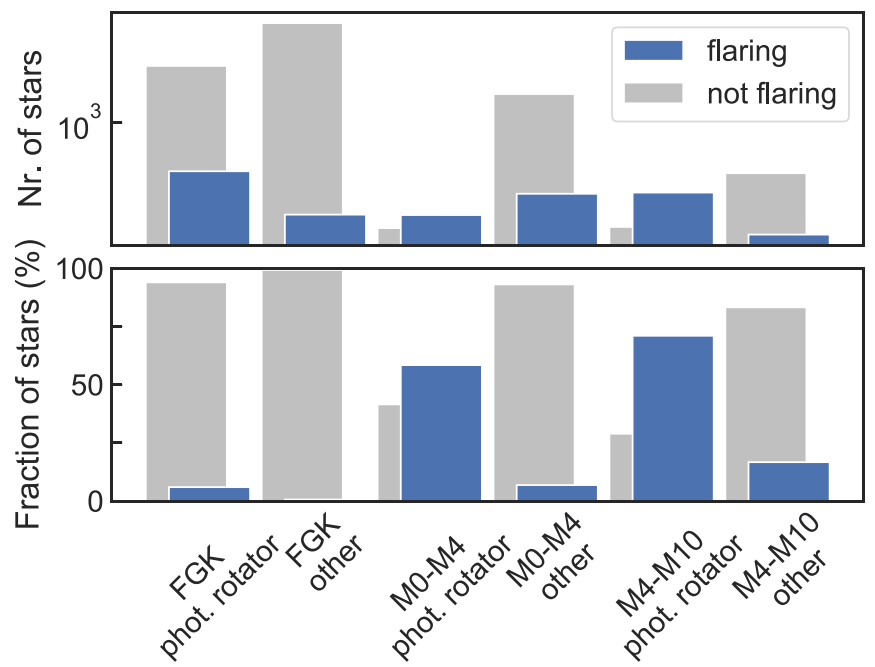

Figure 7. Histograms of the number (upper panel) and fraction (lower panel) of flaring stars (blue) compared to ones for which we do not detect flares (gray). Bins separate the sample into F, G, and K stars; early M dwarfs of type M0 M4; and late M dwarfs of type M4-M10; each of these are further grouped by whether or not a rotation period could be photometrically measured. Flares are detected for $\sim 60 \%$ of fast rotating early and late $\mathrm{M}$ dwarfs in the TESS sample. In contrast, only $\sim 10 \%$ of all other $\mathrm{M}$ dwarfs show detectable flaring.

\subsection{Flares on Fast Rotators}

Stellar rotation is suggested to be linked to the flaring of a star according to the dynamo theory of magnetic field generation (e.g., Moffatt 1978; Parker 1979). The interaction between rotation and convection can cause high magnetic activity (e.g., Browning 2008), whose energy is then released through flares. Two groups of rotators are observed among low-mass stars: fast rotators $\left(P_{\text {rot }}<10\right.$ days) and slow rotators $\left(P_{\text {rot }}>70\right.$ days; e.g., Newton et al. 2016b, 2018). Fast rotating M dwarfs $\left(P_{\text {rot }}<10\right.$ days) exhibit a saturated level of activity, while for slower rotators, the ratio of $\mathrm{X}$-ray, $\mathrm{H} \alpha$, and $\mathrm{Ca} \mathrm{H} \& \mathrm{~K}$ flux to bolometric luminosity declines rapidly (West et al. 2015; Astudillo-Defru et al. 2017; Newton et al. 2017; Wright et al. 2018). The relationship between activity and rotation is typically parameterized in terms of the Rossby number, which seeks to remove the mass dependence by dividing the rotation period by the convective overturn timescale (Noyes et al. 1984). This behavior is consistent across the main sequence from solar-type stars to $\mathrm{M}$ dwarfs and across the fully convective transition at the lowest stellar masses (Newton et al. 2017; Wright et al. 2018).

We here define two categories: "photometric rotator" and "other." We use the phrase "photometric rotator" synonymously to "a star with a photometrically measured rotation period by TESS or KELT." Consequently, rotating stars without a detected modulation are not included in this definition. Our rotation analysis based on TESS data extends to fast rotators with $P_{\text {rot }}<5$ days. The KELT data cover a limited sample of stars with longer rotation periods. Hence, the "other" category might still contain many fast rotators with $P_{\text {rot }}>5$ days, for which we do not have measured rotation periods.

We find that about $60 \%$ of fast rotating early and late $\mathrm{M}$ dwarfs in the TESS sample show flaring that is detectable (Figure 7). In contrast, only $10 \%$ of all "other" $\mathrm{M}$ dwarfs have flares. Among F, G, and $\mathrm{K}$ stars, fewer than $5 \%$ of "photometric rotators" and almost no "others" have flares.

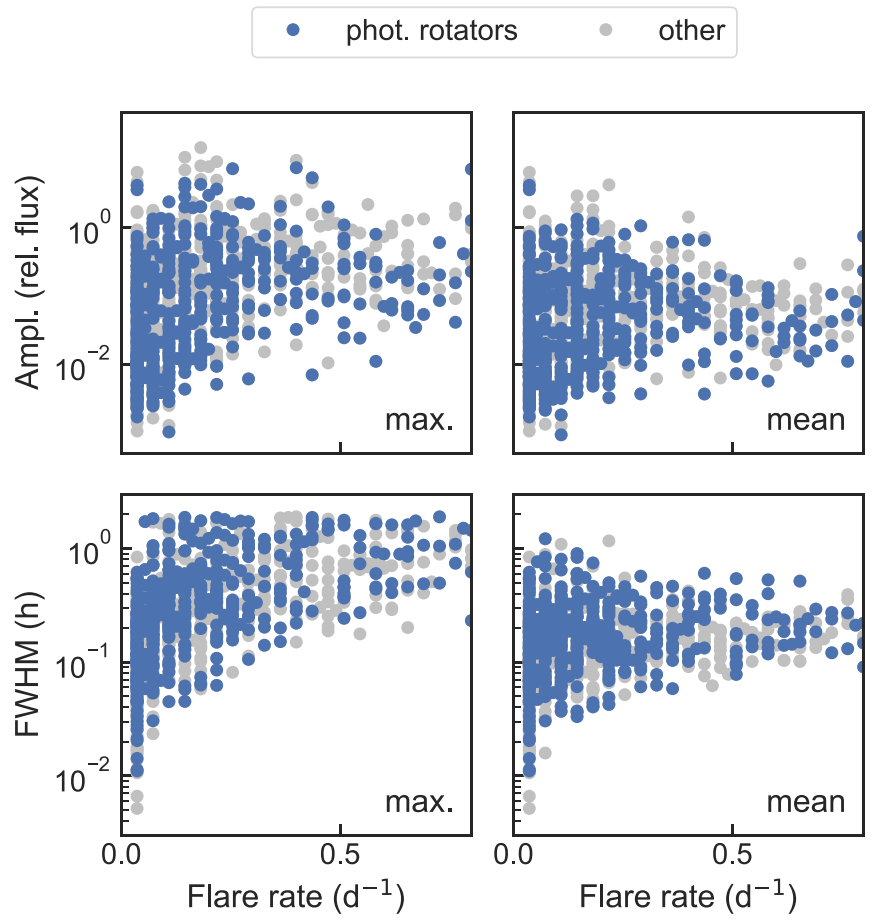

Figure 8. The maximum flare amplitude (upper left), mean flare amplitude (upper right), maximum FWHM (lower left), and mean FWHM (lower right) of each star depending on the flare rate per day. The samples are further separated into stars which have photometrically measured rotation periods (blue) and ones that do not (gray). Stars with higher flare rates have a significantly higher maximum flare amplitude and maximum FWHM. There is no significant difference between photometric rotators and other stars.

This solidifies past findings from, for example, the Kepler (Davenport 2016; Van Doorsselaere et al. 2017) and MEarth (Mondrik et al. 2019) surveys.

Without fast rotation, there might not be enough energy stored in the magnetic field lines to trigger frequent strong flaring. However, in fully convective stars, the dynamo mechanism is not well understood. For stars with a radiative zone and a convective zone, the interface between these two zones is what is believed to power the dynamo (Durney 1993). Lacking this interface, fully convective $\mathrm{M}$ dwarfs (typically with spectral types M4 and later) might generate their magnetic fields through other means (see, e.g., Stassun et al. 2011). Nevertheless, the similar behavior in activity and rotation period (measured via $\mathrm{H} \alpha$, Newton et al. 2017, and X-ray, Wright et al. 2018 data) suggests that the manifestations of the magnetic fields in partially and fully convective objects are similar.

For our sample, it is difficult to disentangle whether the slow rotation impacts both the flare rate and flare amplitude, or just one of them. First, if the flare rate of slow rotators is indeed lower, the 28 day observation span with TESS is not sufficient to detect their flaring. Second, our detection algorithm is not focused on the detection of the smallest flares; slow rotators could still flare frequently, yet at low amplitudes that are not detected.

Moreover, we find that the maximum flare amplitude and FWHM increase for stars with more frequent flaring (Figure 8). This seems to be independent of whether or not a star is a photometric rotator. Furthermore, stars with higher flare rates show similar mean values of their flares amplitudes and durations. 

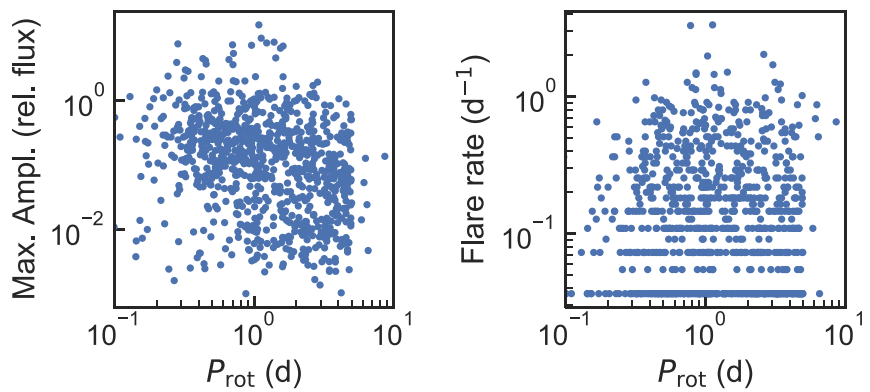

Figure 9. The maximum flare amplitude (left panel) and number of flares per day (right panel) are studied as a function of the rotation periods $P_{\text {rot }}$ measured by TESS and KELT. There is no significant correlation between photometric fast rotators and the amplitude of their superflares, albeit we note that our sample is mostly limited to $P_{\text {rot }}<1$. There are signs that the fastest rotators ( $P_{\text {rot }}<0.3$ days $)$ do not flare as frequently as the rotators with $P_{\text {rot }}>0.3$ days. However, this remains to be reexamined with a larger sample size.

We do not find a correlation between stars' rotation periods and their flare amplitudes (Figure 9). Large flares seem to be as likely to occur on photometric fast rotators as on the other stars in our sample. This is in agreement with previous findings (e.g., Maehara et al. 2012) and can be explained if the magnetic field energy is stored near a starspot. It was shown for different star types that in this scenario, the rotation period does not influence the maximum energy (e.g., Rodono et al. 1986).

Our results suggest a link between the flare frequency and the rotation period for the fastest photometric rotators (Figure 9). Stars with $P<0.3$ days reach only half the maximum flare frequency as those with $P>0.3$ days. However, this could also be a sampling issue; there are fewer photometric rotators with $P<0.3$ days, and the distribution of the flare frequency, peaking at $\sim 0.1$ flares per day, shows a long tail until $\sim 0.5$ flares per day.

For $P>1$ day, the frequency of superflares (flares with energies $>10^{33} \mathrm{erg}$ ) was reported to decrease with period (e.g., Maehara et al. 2012). For our sample, however, the available rotation period information is too sparse to study this link for rotation periods $P>1$ day.

\subsection{Stars Show Flares with Flux Increases of Multiple Magnitudes}

Chang et al. (2018) conducted a study of flaring M-dwarf stars in the Kepler sample. They found eight flares that increase the stellar brightness at peak by a factor of 2 or more. The authors define these events as "hyperflares" (we do not follow this nomenclature here). The strongest flare they detected has a peak luminosity five times the quiescent flux.

The TESS sample profits from the mission's observing strategy, allowing us to identify 99 flaring stars with 140 individual flares that fulfill the Chang et al. (2018) criterion of at least doubling the stellar brightness. A list of these targets can be created from Table 1 by sorting by the amplitude column.

\subsection{The Largest Superflare and the Most Energetic Superflare in the TESS Sample}

260506296 (2MASS J06270005-5622041), an M4.5V dwarf star $(3189 \mathrm{~K})$ with TESS mag 13.7 , shows the largest flare amplitude in our sample (Figure 10). Over the course of $1 \mathrm{hr}$, the star increases its optical brightness by a factor of 16.1, releasing a bolometric energy of $10^{34.7} \mathrm{erg}$. The superflare is
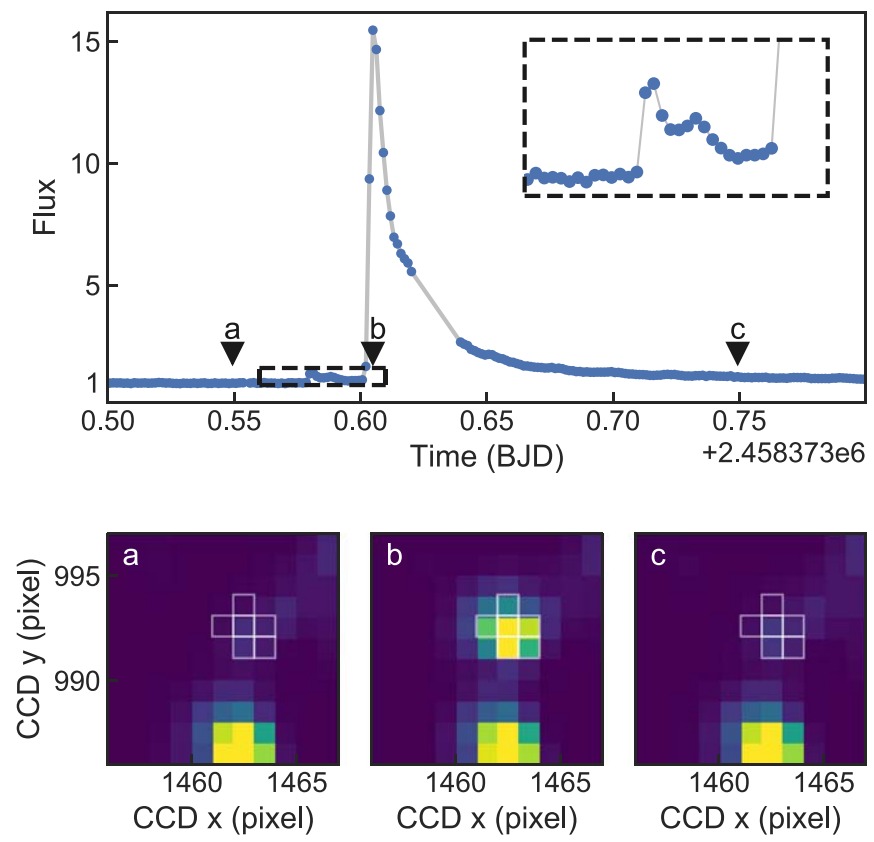

Figure 10. The largest flare in our sample is on 260506296, an M4.5V dwarf star $(3189 \mathrm{~K})$ with TESS mag 13.7. Upper panel: TESS PDC-SAP lightcurve. Lower panel: TESS target pixel images at the times before the flare (a), during the flare peak (b), and after the flare (c), as indicated in the upper panel. Our best-fit model shows that during the peak, the star increases its brightness by a factor of 16.1. This superflare is preceded by a series of smaller flares (inlay). We inspect the target pixel files for this outburst and confirm that the flare originates from the target.

preceded by a series of smaller flares. We inspect the individual target pixel files for this outburst using the lightkurve (Vinícius et al. 2018) module. This confirms that the flare is indeed on TIC 260506296.

In contrast, the most energetic superflare in our sample reaches $10^{36.9} \mathrm{erg}$. It is found on 332487879 (2MASS J23211550-2659121), a G-type giant star $\left(5192 \mathrm{~K}, 9.3 R_{\odot}\right)$ with TESS mag 4.9. Such energetic flares on giant stars are common (e.g., Balona et al. 2015; Van Doorsselaere et al. 2017), which is surprising, as the evolution off the main sequence should decrease their magnetic field (Simon \& Drake 1989). Possible explanations could be that the giant's surface magnetic field is conserved, or that the flare actually originates from an unresolved dwarf star companion. Notably, 332487879's enormous release of energy only leads to a flare amplitude of 1.01. This is a direct consequence of the stellar type, namely effective temperature and radius, following Equations (5)-(9). In comparison, the energy output for the M dwarf TIC 260506296 is significantly lower, despite its much larger amplitude; this is due to the $\mathrm{M}$ dwarf's small radius and lower effective temperature.

\subsection{Flare Frequency Distributions}

We study the flare energies and flare rates as a function of stellar type and rotation period (Figure 11). This is commonly denoted as the flare frequency distribution (FFD; e.g., Gershberg 1972; Lacy et al. 1976; Hawley et al. 2014). The FFD shows the cumulative rate of flares per day, i.e., how often a flare of a certain energy or higher is detected.

Using the TIC information, we separate F, G, and $\mathrm{K}$ stars from early M dwarfs (M0-M4; 3905-3200 K) and late M dwarfs (M4 M10; 3200-2285 K). These are further divided into stars with 

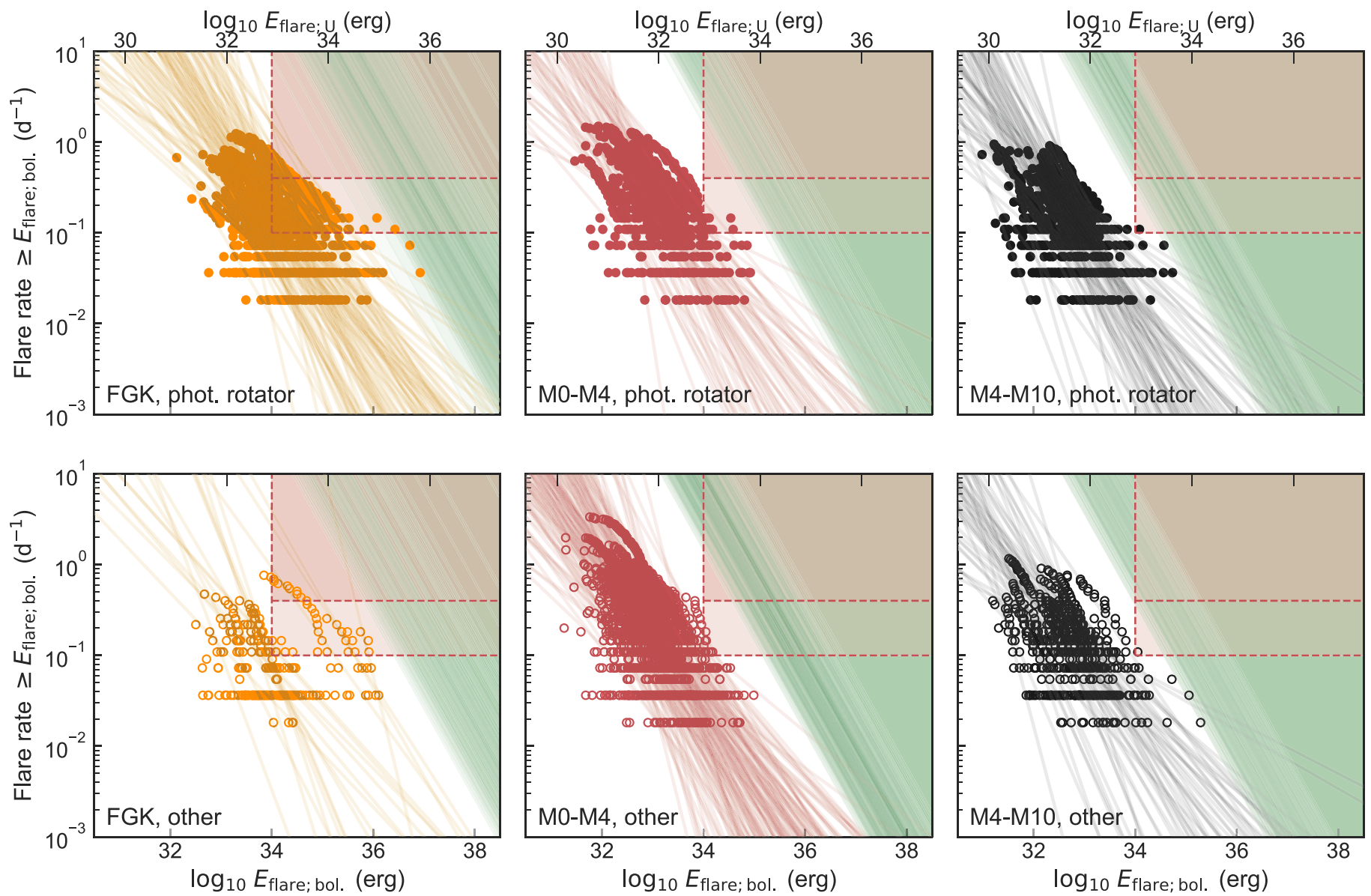

Figure 11. Flare frequency distributions (FFD) in the context of prebiotic chemistry (green area) and ozone sterilization (red areas). The $x$-axis shows the flare energy, as bolometric energy $E_{\mathrm{flare} ; \mathrm{bol}}$ on the lower ticks, and $U$-band energy $E_{\mathrm{flare}, U}$ on the upper ticks. The $y$-axis shows the cumulative rate of flares per day, i.e., how often a flare with at least a certain energy appears. Different panels show F, G, and K stars (orange); early M dwarfs (red); and late M dwarfs (black), separated into photometric rotators (filled circle) and others (unfilled circle). Solid lines are linear fits to the double-logarithmic FFD of each star, extrapolating into regimes that could not be observed. The green area denotes the minimum flare rate and energy required to trigger prebiotic chemistry on a potential exoplanet (expanded from Rimmer et al. 2018; see Section 5.1). The different green shadings show each threshold for each star, which depends on the stellar radius and effective temperature (Equation (10)). In the red shaded region derived from Tilley et al. (2019), intense flares are frequent enough that ozone layers cannot survive and planet surfaces may be sterile (see Section 5.5). We mark two ozone sterilization regions: a permissive threshold for flare rates $\geqslant 0.1$ per day (lighter red area), and a conservative threshold for flare rates $\geqslant 0.4$ per day (darker red area). Fourteen stars, including nine early $\mathrm{M}$ dwarfs and two late $\mathrm{M}$ dwarfs, in the TESS sample fulfill the criteria of prebiotic chemistry. On the other hand, potential exoplanets around 100 stars, including only $15 \mathrm{M}$ dwarfs, might suffer from ozone depletion.

photometrically measured rotation modulation ("phot. rotator") and ones without ("other"; see Section 4.3). Each star's FFD is fit with a line in the $\log -\log$ space to extrapolate the trend, following $\log _{10}$ (flare rate $)=\alpha \log _{10}\left(E_{\text {bol. }}\right)+\beta$. The best-fit parameters $\alpha$ and $\beta$ are included in Table 1.

We find no significant difference between stars with and without detected rotation modulation. This is somewhat surprising given that fast rotating $\mathrm{M}$ dwarfs are suggested to flare more frequently. Moreover, we find that the average flare energy per star depends on the effective temperature and radius of the star, which is a direct consequence of Equations (5)-(9). For F, G, and $\mathrm{K}$ stars, this effect, in addition to their lower contrast (see Section 4.2), is why they generally show higher flare energies than early $\mathrm{M}$ dwarfs and late $\mathrm{M}$ dwarfs.

\section{Discussion}

\subsection{Which Flares Deliver Necessary Energy to Trigger Prebiotic Chemistry?}

We combine the measured flare energies and rates with the laboratory study conducted by Rimmer et al. (2018), who delineate "abiogenesis zones." These are zones outside of which a specific prebiotic chemical scenario cannot succeed around main-sequence stars. This considers ribonucleotides, required for ribonucleic acid (RNA) synthesis, as a starting point for prebiotic chemistry (e.g., Sutherland 2015). The authors consider the competition between reactions that produce ribonucleotides in the presence of UV light (200-280 nm), with other bimolecular reactions that produce inert adducts with no prebiotic interest. They calculate the UV light from a flare multiplied by the flare frequency and compare it to the lifetime of prebiotic intermediates required for ribonucleotide formation (e.g., Xu et al. 2018). Rimmer et al. (2018) develop equations for a planet at a fixed distance from its host star. Here, we adjust the star-planet distance, $a$, to keep the planet at a distance from its host star so that the flux is the same as 1 Earth flux (as defined by Kane et al. 2016). The flare frequency needed to drive the prebiotic chemistry, $\nu$, is a function of the flare's $U$ band energy $E_{U}$, the stellar radius, $R_{*}$, and the stellar temperature, $T_{*}$. Then, $\nu$ can be derived following

$$
\nu \geqslant 25.5 \text { day }^{-1}\left(\frac{10^{34} \mathrm{erg}}{E_{U}}\right)\left(\frac{R_{*}}{R_{\odot}}\right)^{2}\left(\frac{T_{*}}{T_{\odot}}\right)^{4} .
$$


To apply this equation to the bolometric energies we derived, we first calculate the flares' $U$-band energy from the bolometric energy. This uses the $U$-band spectral response function and assumes a $9000 \mathrm{~K}$ blackbody for the flare. We find that $7.6 \%$ of the flare's bolometric energy falls into the $U$ band, leading to $E_{U} \approx 7.6 \% E_{\mathrm{bol}}$.

Flare frequencies satisfying Equation (10) could drive the synthesis of pyrimidine ribonucleotides, the building blocks of RNA, from hydrogen cyanide and bisulfite in liquid water. These flare rates establish a necessary condition for the origin of life's building blocks according to the scenario proposed by $\mathrm{Xu}$ et al. (2018). Having the necessary UV light from stellar activity is not a sufficient condition for this scenario. Liquid water, hydrogen cyanide, bisulfite, and other feedstock molecules are also necessary.

Figure 11 displays the flare rates of the stars in our sample, compared to this inequality. We find that 14 stars have enough flaring (at their current age) to deliver the UV energy at a rate that could trigger prebiotic chemistry on a potential exoplanet. These include nine early $M$ dwarfs and two late $M$ dwarfs. Out of these, zero early $\mathrm{M}$ dwarfs are brighter than 12th TESS magnitude. A list of the targets whose flaring have this potential can be created from Table 1 by sorting by the "prebiotic chemistry" column.

It is important to note that most of the stars in our sample are likely old (albeit we currently have limited knowledge of their exact ages). The onset of prebiotic chemistry likely occurred during earlier stages of exosolar systems, when the stars were younger. Given that young $\mathrm{M}$ dwarfs were more active (West et al. 2008) and thus assuming that their flaring was more frequent and energetic in the past, our results provide a lower limit. If a star's FFD passes this cutoff now, it will also have done so in a younger stage.

\subsection{The Impact of CMEs}

A CME often follows a stellar flare and can have a substantial impact on potential exoplanets. A CME is a large release of plasma and the connected magnetic field from the stellar corona.

To estimate the impact of CMEs, we apply the empirical relationship between flare energy and CME mass found by Aarnio et al. (2012). This has been calibrated against observations of flares and CMEs from the Sun, as well as a sample of well-studied, very active pre-main-sequence stars. The calibration encompasses flare energies from $\sim 10^{28} \mathrm{erg}$ up to $\sim 10^{38}$ erg-comparable to the strongest flares included in this study - and which correspond to CME masses of up to $\sim 10^{22}$ g (Aarnio et al. 2011, 2012).

This empirical relation, however, is applicable to the flare energy that is emitted in X-rays, whereas in this work we determined the flare energy that is emitted bolometrically (Section 3.7). To apply it to our data, we estimate the X-ray flare energy as $1 \%$ of the bolometric energy, which is based on findings for the strongest solar flares. These have X-ray fluxes of about $10^{-4} \mathrm{~W} \mathrm{~m}^{-2}$ (Aarnio et al. 2011), corresponding to $\mathrm{X}$-ray energies of $10^{29} \mathrm{erg}$ (Aarnio et al. 2012) and to bolometric energies of $10^{31} \mathrm{erg}$ (Maehara et al. 2015). Hence, for the same flare strength, the energy emitted bolometrically is typically larger by a factor of 100 (see also Osten \& Wolk 2015).

Thus, we adapt the following equation from Aarnio et al. (2012) for the CME mass, $M_{\mathrm{CME}}$, in terms of the bolometric flare energy:

$$
M_{\mathrm{CME}}=(2.7 \pm 1.2) \times\left(\frac{E_{\mathrm{flare} ; \mathrm{bol}}}{100}\right)^{(0.63 \pm 0.04)} .
$$

All values are in cgs units.

From Equation (11), we find a median CME mass of $10^{20.0} \mathrm{~g}$, ranging from a minimum of $10^{18.5}$ to $10^{22.3} \mathrm{~g}$ for the most massive CME. This could have accompanied the strongest flare in our sample, which was found on the giant star $332487879\left(5192 \mathrm{~K}, 9.3 R_{\odot}\right)$. All estimates are also given in Tables 1 and 2.

CME events mainly happen along a given direction and, as such, are nonisotrop events. Their impact depends among other factors on the semimajor axis and inclination of the exoplanet's orbit (Kay et al. 2016). As exoplanets' habitable zones around $\mathrm{M}$ dwarfs are close to their star, the probability of being hit by a CME is higher than for their counterparts around Sun-like stars. Even though the overall chance of a strong CME hitting an exoplanet is relatively low, it is interesting to consider the potential impact on habitability. CMEs impinging on a nitrogen-rich atmosphere can be useful for prebiotic chemistry via the efficient production of hydrogen cyanide, and possibly for atmospheric warming, via the production of nitrous oxide (Airapetian et al. 2016). On the other hand, CMEs can contribute significantly to the potential loss or transformation of exoplanet atmospheres through ion pick up (Lammer et al. 2007; Cohen et al. 2015). For M dwarfs, the exoplanet's magnetic field has to be up to hundreds of Gauss strong to shield from atmospheric loss through CMEs, making them a more detrimental factor than stellar winds (Kay et al. 2016). Very energetic particles from the CME may produce secondary particles that can reach the surface, changing mutation rates for life there (Smith et al. 2004).

\subsection{Ozone Depletion}

The radiation from stellar proton events (SPEs) could deleteriously impact the habitability of exoplanets. However, Atri (2017) find that while energetic SPEs could trigger extinction events among complex life, SPEs are unlikely to lead to surface sterilization. In particular, exoplanets with magnetic fields and/or substantial atmospheres are sufficiently shielded from SPEs.

The impact of protons and photons from M-dwarf stellar flares on the atmosphere of a modern-Earth analog exoplanet has been modeled by Tilley et al. (2019; see also, e.g., Youngblood et al. 2017). They find that the impact of flares of energy $10^{34} \mathrm{erg}$ at a frequency of $1 \mathrm{month}^{-1}$ or greater will result in the removal of $99.99 \%$ or more of the ozone layer, incidentally admitting potentially sterilizing doses of UV radiation to the planet surface. Tilley et al. (2019) estimate that, conservatively, 0.083 of M-dwarf flares will strike a habitable-zone exoplanet, meaning that terrestrial-type ozone layers should not persist on stars with flares of $\geqslant 10^{34} \mathrm{erg}$ at a frequency of $\geqslant 0.4$ day $^{-1}$. A more permissive limit could be drawn at $\geqslant 0.1$ day $^{-1}$. These parameter spaces are marked in red in Figure 11.

Few of the stars we consider in this study fall into this parameter space. For the permissive limit of $0.1 \mathrm{day}^{-1}$, potential exoplanets around 100 stars, including $15 \mathrm{M}$ dwarfs, might suffer from ozone depletion. For the conservative limit of 0.4 day $^{-1}, 22$ stars could be affected. However, it is possible that more frequent but smaller flares and/or less frequent but 
more energetic flares may also remove ozone layers. Further modeling work is required to rule on this possibility. We note that even in the absence of an ozone layer, life can survive in the ocean or the subsurface, meaning that flares may not be a strict barrier to habitability.

From a different point of view, ozone has been proposed as a biosignature (Segura et al. 2003). Exoplanets subject to sufficiently intense flares would not be able to develop ozone layers, leading to a potential "false negative" scenario for ozone. Other biosignatures may be similarly affected. The FFDs we derive in this paper will enable detailed modeling of the atmospheric states of planets orbiting these stars and will enable the assessment of false-positive and false-negative scenarios for a biosignature search for these objects.

\subsection{Additional Constraints for Habitability}

We discussed selected aspects of how flare events can influence an exoplanet's habitability: how flares could trigger prebiotic chemistry (Section 5.1), the impact of CMEs accompanied with flares (Section 5.2), and ozone depletion (Section 5.3).

A more comprehensive view of habitability should also consider the following factors: the exoplanet's orbit (semimajor axis and eccentricity), the stellar type, stellar companions, the exoplanet's atmospheric composition or absence of an atmosphere, the exoplanet's surface, potential subsurface habitability, and many other factors (see, e.g., Seager 2013; Barnes et al. 2015; Shields et al. 2016; Meadows \& Barnes 2018).

\subsection{On the Habitability around TIC 260506296 and Other Superflare M Dwarfs}

One of the most interesting targets for habitable exoplanet search will be $\mathrm{M}$ dwarfs whose FFDs fill the lower-right corner of the panels in Figure 11. In these areas, the flaring is energetic enough to trigger prebiotic chemistry (green area), but not with such a large frequency that would lead to ozone depletion (red areas). This is an particularly interesting parameter space for $\mathrm{M}$ dwarfs, which can soon be explored further with continuous one-year observations in the TESS continuous viewing zone. Note, however, that we here assume the FFDs can be extrapolated in this regime; findings from Kepler suggest that this might not always be the case for M dwarfs (e.g., Davenport 2016).

What can we say about TIC 260506296, which shows the largest superflare in the sample? Its flux increases by a factor of 16.1 in the TESS band over the course of $1 \mathrm{hr}$, releasing a bolometric energy of $10^{34.7} \mathrm{erg}$ (see Section 4.5). In contrast, the Carrington event on our Sun $(\mathrm{G} 2 \mathrm{~V}$; $5772 \mathrm{~K})$ released $10^{32} \mathrm{erg}$. Yet, the flare energy of these events on $\mathrm{M}$ dwarfs depend on the quiescent flux. Taking the radius and temperature differences between $\mathrm{M}$ dwarfs and the Sun into account (Equations (5)-(9)), it becomes clear how much more extreme flares are on these prime targets for exoplanet missions.

The superflare on 260506296 is comparable to, yet smaller than, the largest superflare reported for Proxima Centauri by Howard et al. (2018). The Proxima Centauri superflare temporarily increased the star's brightness by a factor of at least 38, releasing $10^{33.5} \mathrm{erg}$. Interestingly, 260506296 $(\mathrm{M} 4.5 \mathrm{~V}, 3189 \mathrm{~K})$ and Proxima Centauri (M5.5V, $3050 \mathrm{~K})$ are comparable stars. Such large stellar flares have been suggested to significantly impact the habitability of putative planetary companions (e.g., Howard et al. 2018).

We find that 260506296 indeed provides substantial flaring to trigger prebiotic chemistry on nearby worlds (see Tables 1 and 2). Its superflare was potentially accompanied by a CME event of $10^{23} \mathrm{~g}$, and the star's FFD does not fall into the regime of ozone depletion by SPEs. All of this is opening interesting avenues for future studies on this potential exoplanet host, as well as other comparable $\mathrm{M}$ dwarfs.

\subsection{Four TESS Exoplanet Candidates Orbiting Flaring $M$ Dwarfs}

Whether we can actually detect exoplanets around such strongly flaring stars is a separate matter. A large fraction of them are fast rotators with high flare frequencies, which can inhibit the detection of small planets (see, e.g., Berta et al. 2012; Kipping et al. 2017). Additionally, planetary mass measurements can be hindered if the stellar rotation and planetary orbital periods are similar or harmonics. This is particularly a problem for habitable-zone planets around fieldaged early M dwarfs (Newton et al. 2016a; Vanderburg et al. 2016).

We do not find any known exoplanets among our flare stars when cross-matching Table 2 with the known exoplanet lists provided by Stephen Kane ${ }^{25}$ and John Southworth ${ }^{26}$ for the TESS Guest Observer program.

We do, however, find four matches among the current TESS alerts, ${ }^{27}$ which could potentially be exoplanets transiting flaring stars from our sample:

1. TIC 32090583 is an $\mathrm{M}$ dwarf with a $4.84 R_{\oplus}$ exoplanet candidate on a 0.438 day orbit.

2. TIC 70797900 is an early $M$ dwarf hosting a singletransit candidate with unknown period. However, its large candidate radius of $17.06 R_{\oplus}$ and $\mathrm{V}$-shaped transit could indicate an eclipsing binary scenario.

3. TIC 206609630 is potentially an M dwarf with a $5.51 R_{\oplus}$ exoplanet candidate on a 0.335 day orbit (note that it has a Gaia source duplication flag).

4. TIC 272086159 is a mid M dwarf with a $9.77 R_{\oplus}$ exoplanet candidate transiting every 16.156 days.

These exosolar systems, if confirmed, could allow interesting case studies. However, the four candidates themselves likely will have thick gas envelopes, increasing the temperature beneath the atmosphere to a level that is too high for liquid water and a solid surface. Nevertheless, potential planetary companions or exomoons (see, e.g., Teachey \& Kipping 2018) might provide the necessary conditions.

\section{Conclusion}

We detect and analyze stellar flares in the short-cadence (2 minutes) lightcurves from the first TESS data release. To do so, we develop a flare-detection pipeline, whose candidates we visually inspect to create a vetted flare candidate list. We then apply our newly developed allesfitter software to fit the profiles of each flare with different models, ranging from pure

\footnotetext{
${ }^{25}$ https://heasarc.gsfc.nasa.gov/docs/tess/data/approved-programs/G011183. txt, online 2018 December 28 .

${ }^{26}$ https://heasarc.gsfc.nasa.gov/docs/tess/data/approved-programs/G011112. txt, online 2018 December 28

27 https://archive.stsci.edu/prepds/tess-data-alerts/, online 2018 December 28.
} 
noise to complex flare sequences. Using nested sampling, we compute the Bayesian evidence of each model. This allows us to robustly select the favored scenario.

We find 1228 flaring stars in the first two TESS sectors, with a total count of 8695 flares. The largest-amplitude flare appears on the M dwarf 260506296 and increases the brightness by a factor of 16.1. The flare with the highest energy output is on the G-type giant star 332487879 , releasing $10^{36.9}$ erg and could be accompanied by a CME of $10^{22.1} \mathrm{~g}$.

Among all observed stars, flares appear on $30 \%$ of mid to late $\mathrm{M}$ dwarfs, on $5 \%$ of early $\mathrm{M}$ dwarfs, and on less than $1 \%$ of F, G, and K stars. Of all flaring stars, 673 are early to late M dwarfs, highlighting that TESS explores an important parameter space for flare studies. In total, we find 531 early $\mathrm{M}$ dwarfs and 142 late M dwarfs.

Moreover, we investigate flaring as a function of photometrically measured stellar rotation periods ("photometric rotator"). A total of $60 \%$ of fast rotating M dwarfs are flare stars. Of the $\mathrm{M}$ dwarfs without detected rotation periods, only $10 \%$ flare. We further find that star's with higher flare rates also have an increased maximum flare amplitude and FWHM. Photometric rotators and other stars show comparable flare rates, amplitudes, and energies; however, we note that our rotation information mainly covers the regime of $P_{\text {rot }}<5$ days. Among photometric rotators, there is a tentative decrease of the flare rate for $P_{\text {rot }}<0.3$ days.

We analyze the FFDs in the context of prebiotic chemistry, CMEs, and ozone depletion. On the one hand, flares have been suggested to deliver the required ultraviolet energy to trigger biogenesis on exoplanets. We find 14 stars, including $11 \mathrm{M}$ dwarfs, which could fulfill the criteria of a minimum flare rate and energy. Nevertheless, most stars do not seem to be able to provide the necessary ultraviolet energy through their flares alone. On the other hand, CMEs and SPEs associated with flares could further impact existing life, with SPEs potentially causing ozone depletion for exoplanet's atmospheres. We find that potential exoplanets around up to 100 stars might suffer from this effect. Notably, these are mostly F-, G-, and K-type stars, while only up to $100 \mathrm{M}$ dwarfs seem affected. This can negatively impact habitability and the search for biosignatures.

Four flaring M dwarfs host exoplanet candidates from recent TESS alerts: TIC 32090583, TIC 70797900, TIC 206609630, and TIC 272086159. Together with other M dwarfs, such as the superflaring 260506296, these systems can open an interesting avenue for future studies of habitability.

It is important to note that these findings alone do not allow ruling on the possibility of life on potential exoplanets. Our study provides novel methods and insights to derive an overview. Yet, when addressing complex topics such as exoplanet habitability, any interesting individual system deserves a detailed study to consider a variety of interrelated factors. Fortunately, TESS will continue delivering these prime targets. Those in the mission's continuous viewing zone in particular will provide reliable statistics on the largest, and potentially rarest, superflares.

We thank Ward Howard for engaging discussions about stellar flares. Funding for the TESS mission is provided by NASA's Science Mission directorate. This paper includes data collected by the TESS mission, which are publicly available from the Mikulski Archive for Space Telescopes (MAST). M. N.G. acknowledges support from MIT's Kavli Institute as a
Torres postdoctoral fellow. T.D. acknowledges support from MIT's Kavli Institute as a Kavli postdoctoral fellow. P.B.R. acknowledges support from the Simons Foundation (SCOL awards 599634). This work was supported in part by grants from the Simons Foundation (SCOL grant \# 495062 to S.R.). M.H.K. acknowledges Allan R. Schmitt and Troy Winarski for making their lightcurve softwares freely available. This research has made use of IMCCE's SkyBoT VO tool.

Facility: TESS.

Software: PYTHON (van Rossum 1995), NUMPY (van der Walt et al. 2011), SCIPY (Jones et al. 2001), MATPLOTLIB (Hunter 2007), TQDM (da Costa-Luis et al. 2018), SEABORN (Waskom et al. 2018), ALLESFITTER (Günther \& Daylan 2019, and M. N. Günther \& T. Daylan 2019, in preparation), ELLC (Maxted 2016), AFLARE (Davenport et al. 2014), DYNESTY (Speagle 2019), EMCEE (Foreman-Mackey et al. 2013), CELERITE (Foreman-Mackey et al. 2017), CORNER (ForemanMackey 2016).

\section{ORCID iDs}

Maximilian N. Günther (iD https://orcid.org/0000-00023164-9086

Zhuchang Zhan (1) https://orcid.org/0000-0002-4142-1800

Paul B. Rimmer (D) https://orcid.org/0000-0002-7180-081X

Sukrit Ranjan (D) https://orcid.org/0000-0002-5147-9053

Keivan G. Stassun (iD https://orcid.org/0000-0002-3481-9052

Ryan J. Oelkers (10 https://orcid.org/0000-0002-0582-1751

Tansu Daylan (iD https://orcid.org/0000-0002-6939-9211

Elisabeth Newton (i) https://orcid.org/0000-0003-4150-841X

Katalin Olah (iD https://orcid.org/0000-0003-3669-7201

Edward Gillen (10 https://orcid.org/0000-0003-2851-3070

Saul Rappaport (i) https://orcid.org/0000-0003-3182-5569

Roland K. Vanderspek (iD https://orcid.org/0000-00016763-6562

David W. Latham (iD https://orcid.org/0000-0001-9911-7388

Joshua N. Winn (iD https://orcid.org/0000-0002-4265-047X

Jon M. Jenkins (iD https://orcid.org/0000-0002-4715-9460

Ana Glidden (iD https://orcid.org/0000-0002-5322-2315

Michael Fausnaugh (1) https://orcid.org/0000-0002-9113-7162

Samuel N. Quinn (D) https://orcid.org/0000-0002-8964-8377

\section{References}

Aarnio, A. N., Matt, S. P., \& Stassun, K. G. 2012, ApJ, 760, 9

Aarnio, A. N., Stassun, K. G., Hughes, W. J., \& McGregor, S. L. 2011, SoPh, 268,195

Airapetian, V. S., Glocer, A., Gronoff, G., Hébrard, E., \& Danchi, W. 2016, NatGe, 9, 452

Airapetian, V. S., Jackman, C. H., Mlynczak, M., Danchi, W., \& Hunt, L. 2017, NatSR, 7, 14141

Anglada-Escudé, G., Amado, P. J., Barnes, J., et al. 2016, Natur, 536, 437

Astudillo-Defru, N., Delfosse, X., Bonfils, X., et al. 2017, A\&A, 600, A13

Atri, D. 2017, MNRAS, 465, L34

Balona, L. A., Broomhall, A.-M., Kosovichev, A., et al. 2015, MNRAS, 450, 956

Barnes, R., Meadows, V. S., \& Evans, N. 2015, ApJ, 814, 91

Benz, A. O., \& Güdel, M. 2010, ARA\&A, 48, 241

Berta, Z. K., Irwin, J., Charbonneau, D., Burke, C. J., \& Falco, E. E. 2012, AJ, 144,145

Berthier, J., Carry, B., Vachier, F., Eggl, S., \& Santerne, A. 2016, MNRAS, 458, 3394

Berthier, J., Vachier, F., Thuillot, W., et al. 2006, in ASP Conf. Ser. 351, Astronomical Data Analysis Software and Systems XV, ed. C. Gabriel et al. (San Francisco, CA: ASP), 367

Björn, L. O. (ed.) 2015, Photobiology: The Science of Light and Life (New York: Springer), 415 
Borucki, W. J., Koch, D., Basri, G., et al. 2010, Sci, 327, 977

Browning, M. K. 2008, ApJ, 676, 1262

Bryce, C. C., Horneck, G., Rabbow, E., Edwards, H. G., \& Cockell, C. S. 2015, IJAsB, 14, 115

Carrington, R. C. 1859, MNRAS, 20, 13

Chang, H. Y., Lin, C. L., Ip, W. H., et al. 2018, ApJ, 867, 78

Cohen, O., Ma, Y., Drake, J. J., et al. 2015, ApJ, 806, 41

Covey, K. R., Hawley, S. L., Bochanski, J. J., et al. 2008, AJ, 136, 1778

Cullum, J., \& Stevens, D. P. 2016, PNAS, 113, 4278

Cullum, J., Stevens, D., \& Joshi, M. 2014, AsBio, 14, 645

da Costa-Luis, C., Stephen, S., Mary, H., et al. 2018, tqdm/tqdm:tqdm v4.28.1 stable, v4.28.1, Zenodo, doi:10.5281/zenodo.1468033

Davenport, J. R. A. 2016, ApJ, 829, 23

Davenport, J. R. A., Hawley, S. L., Hebb, L., et al. 2014, ApJ, 797, 122

Diaz, B., \& Schulze-Makuch, D. 2006, AsBio, 6, 332

Dittmann, J. A., Irwin, J. M., Charbonneau, D., et al. 2017, Natur, 544, 333

Dole, S. 1964, Habitable Planets for Man (Waltham, MA: Blaisdell)

Doyle, J., Shetye, J., Antonova, A., et al. 2018, MNRAS, 475, 2842

Durney, B. R. 1993, ApJ, 407, 367

Estrela, R., \& Valio, A. 2018, AsBio, 18, 1414

Foreman-Mackey, D. 2016, JOSS, 24, 1

Foreman-Mackey, D., Agol, E., Ambikasaran, S., \& Angus, R. 2017, celerite: Scalable 1D Gaussian Processes in $\mathrm{C}++$, Python, and Julia, Version 0.3.1, Astrophysics Source Code Library, ascl:1709.008

Foreman-Mackey, D., Hogg, D. W., Lang, D., \& Goodman, J. 2013, PASP, 125,306

France, K., Parke Loyd, R. O., Youngblood, A., et al. 2016, ApJ, 820, 89

Gershberg, R. E. 1972, Ap\&SS, 19, 75

Gillon, M., Triaud, A. H. M. J., Demory, B.-O., et al. 2017, Natur, 542, 456

Grenfell, J. L., Grießmeier, J.-M., von Paris, P., et al. 2012, AsBio, 12, 1109

Günther, M. N., \& Daylan, T. 2019, allesfitter: Flexible Star and Exoplanet Inference from Photometry and Radial Velocity, Version 0.9.8, Astrophysics Source Code Library, ascl:1903.003

Günther, M. N., \& Daylan, T. 2019, MNGuenther/allesfitter, v0.9.8, Zenodo, doi: 10.5281 /zenodo. 3552952

Hawley, S. L., Davenport, J. R. A., Kowalski, A. F., et al. 2014, ApJ, 797, 121

Hawley, S. L., \& Pettersen, B. R. 1991, ApJ, 378, 725

Henry, T. J., Kirkpatrick, J. D., \& Simons, D. A. 1994, AJ, 108, 1437

Hodgson, R. 1859, MNRAS, 20, 15

Howard, W. S., Tilley, M. A., Corbett, H., et al. 2018, ApJL, 860, L30

Huang, S.-S. 1959, AmSci, 47, 397

Hunter, J. D. 2007, CSE, 9, 90

Jackman, J. A. G., Wheatley, P. J., Pugh, C. E., et al. 2018, MNRAS, 477, 4655

Jackman, J. A. G., Wheatley, P. J., Pugh, C. E., et al. 2019, MNRAS, 482, 5553

Jenkins, J. M. 2002, ApJ, 575, 493

Jenkins, J. M., Chandrasekaran, H., McCauliff, S. D., et al. 2010, Proc. SPIE, 7740, 77400D

Jenkins, J. M., Twicken, J. D., McCauliff, S., et al. 2016, Proc. SPIE, 9913, 99133E

Jenkins, J. M. 2017, Kepler Data Processing Handbook: Overview of the Science Operations Center, Kepler Science Document, KSCI-19081-002

Jones, E., Oliphant, T., Peterson, P., et al. 2001, SciPy: Open Source Scientific Tools for Python, Version 1.3.1, http://www.scipy.org/

Kaltenegger, L., \& Traub, W. A. 2009, ApJ, 698, 519

Kane, S. R., Hill, M. L., Kasting, J. F., et al. 2016, ApJ, 830, 1

Kass, R. E., \& Raftery, A. E. 1995, J. Am. Stat. Assoc., 90, 773

Kasting, J. F., Kopparapu, R., Ramirez, R. M., \& Harman, C. E. 2014, PNAS, 111,12641

Kasting, J. F., Whitmire, D. P., \& Reynolds, R. T. 1993, Icar, 101, 108

Kay, C., Opher, M., \& Kornbleuth, M. 2016, ApJ, 826, 195

Kiang, N. Y., Segura, A., Tinetti, G., et al. 2007, AsBio, 7, 252

Kipping, D. M., Cameron, C., Hartman, J. D., et al. 2017, AJ, 153, 93

Kopparapu, R. K., Ramirez, R., Kasting, J. F., et al. 2013, ApJ, 765, 131

Kopparapu, R. K., Ramirez, R. M., SchottelKotte, J., et al. 2014, ApJL, 787, L29

Kowalski, A. F., Hawley, S. L., Hilton, E. J., et al. 2009, AJ, 138, 633

Kowalski, A. F., Hawley, S. L., Wisniewski, J. P., et al. 2013, ApJS, 207, 15

Lacy, C. H., Moffett, T. J., \& Evans, D. S. 1976, ApJS, 30, 85

Lammer, H., Lichtenegger, H. I. M., Kulikov, Y. N., et al. 2007, AsBio, 7, 185

Law, N. M., Fors, O., Ratzloff, J., et al. 2015, PASP, 127, 234

Lingam, M., \& Loeb, A. 2017, ApJ, 848, 41

Lomb, N. R. 1976, Ap\&SS, 39, 447

Maehara, H., Shibayama, T., Notsu, S., et al. 2012, Natur, 485, 478

Maehara, H., Shibayama, T., Notsu, Y., et al. 2015, EP\&S, 67, 59

Maxted, P. F. L. 2016, A\&A, 591, A111

Meadows, V. S., \& Barnes, R. K. 2018, in Handbook of Exoplanets, ed. H. Deeg \& J. Belmonte (Cham: Springer), 57
Moffatt, H. K. 1978, Magnetic Field Generation in Electrically Conducting Fluids (Cambridge: Cambridge Univ. Press)

Mondrik, N., Newton, E., \& Irwin, D. C. J. 2019, ApJ, 870, 10

Mullan, D. J., \& Bais, H. P. 2018, ApJ, 865, 101

Newton, E. R., Irwin, J., Charbonneau, D., Berta-Thompson, Z. K., \& Dittmann, J. A. 2016a, ApJL, 821, L19

Newton, E. R., Irwin, J., Charbonneau, D., et al. 2016b, ApJ, 821, 93

Newton, E. R., Irwin, J., Charbonneau, D., et al. 2017, ApJ, 834, 85

Newton, E. R., Mondrik, N., Irwin, J., Winters, J. G., \& Charbonneau, D. 2018 AJ, 156, 217

Noyes, R. W., Hartmann, L. W., Baliunas, S. L., Duncan, D. K., \& Vaughan, A. H. 1984, ApJ, 279, 763

Nutzman, P., \& Charbonneau, D. 2008, PASP, 120, 317

O’Malley-James, J. T., \& Kaltenegger, L. 2018, MNRAS, 481, 2487

Oelkers, R. J., Rodriguez, J. E., Stassun, K. G., et al. 2018, AJ, 155, 39

Osten, R. A., \& Wolk, S. J. 2015, ApJ, 809, 79

Pál, A., Molnár, L., \& Kiss, C. 2018, PASP, 130, 114503

Parker, E. N. 1979, Cosmical Magnetic Fields. Their Origin and Their Activity (Oxford: Clarendon Press)

Parnell, C. E., \& Jupp, P. E. 2000, ApJ, 529, 554

Pecaut, M. J., \& Mamajek, E. E. 2013, ApJS, 208, 9

Pierrehumbert, R., \& Gaidos, E. 2011, ApJL, 734, L13

Ramirez, R. M., \& Kaltenegger, L. 2014, ApJL, 797, L25

Ranjan, S., Wordsworth, R., \& Sasselov, D. D. 2017, ApJ, 843, 110

Reid, I. N., Cruz, K. L., Allen, P., et al. 2004, AJ, 128, 463

Ricker, G. R., Winn, J. N., Vanderspek, R., et al. 2014, Proc. SPIE, 9143, 914320

Rimmer, P. B., Xu, J., Thompson, S. J., et al. 2018, SciA, 4, eaar3302

Rodono, M., Cutispoto, G., Pazzani, V., et al. 1986, A\&A, 165, 135

Scalo, J., Kaltenegger, L., Segura, A. G., et al. 2007, AsBio, 7, 85

Scargle, J. D. 1982, ApJ, 263, 835

Seager, S. 2013, Sci, 340, 577

Segura, A., Krelove, K., Kasting, J. F., et al. 2003, AsBio, 3, 689

Segura, A., Walkowicz, L. M., Meadows, V., Kasting, J., \& Hawley, S. 2010 AsBio, 10, 751

Shibata, K., \& Takasao, S. 2016, ASSL, 427, 373

Shibayama, T., Maehara, H., Notsu, S., et al. 2013, ApJS, 209, 5

Shields, A. L., Ballard, S., \& Johnson, J. A. 2016, PhR, 663, 1

Simon, T., \& Drake, S. A. 1989, ApJ, 346, 303

Smith, D. S., Scalo, J., \& Wheeler, J. C. 2004, Icar, 171, 229

Smith, J. C., Stumpe, M. C., Van Cleve, J. E., et al. 2012, PASP, 124, 1000 Speagle, J. S. 2019, arXiv:1904.02180

Stassun, K. G., Hebb, L., Covey, K., et al. 2011, in ASP Conf. Ser. 448, 16th Cambridge Workshop on Cool Stars, Stellar Systems, and the Sun, ed. C. Johns-Krull, M. K. Browning, \& A. A. West (San Francisco, CA: ASP), 505 Stellingwerf, R. F. 1978, ApJ, 224, 953

Stelzer, B., Damasso, M., Scholz, A., \& Matt, S. P. 2016, MNRAS, 463, 1844 Stetson, P. B. 1996, PASP, 108, 851

Stumpe, M. C., Smith, J. C., Catanzarite, J. H., et al. 2014, PASP, 126, 100

Sutherland, J. 2015, in European Planetary Science Congress, ed. G. Choblet et al. (Göttingen: Copernicus), EPSC2015-1

Szabó, R., Sárneczky, K., Szabó, G. M., et al. 2015, AJ, 149, 112

Teachey, A., \& Kipping, D. M. 2018, SciA, 4, eaav1784

Tilley, M. A., Segura, A., Meadows, V., Hawley, S., \& Davenport, J. 2019, AsBio, 19, 64

van der Walt, S., Colbert, S. C., \& Varoquaux, G. 2011, CSE, 13, 22

Van Doorsselaere, T., Shariati, H., \& Debosscher, J. 2017, ApJS, 232, 26

van Rossum, G. 1995, Python Tutorial, Tech. Rep. CS-R9526, Centrum voor Wiskunde en Informatica (CWI), Amsterdam

Vanderburg, A., Plavchan, P., Johnson, J. A., et al. 2016, MNRAS, 459, 3565

Venot, O., Rocchetto, M., Carl, S., Roshni Hashim, A., \& Decin, L. 2016, ApJ, 830,77

Vinícius, Z., Barentsen, G., Hedges, C., Gully-Santiago, M., \& Cody, A. M. 2018, KeplerGO/lightkurve, Version 1.5.1, Zenodo, doi:10.5281/zenodo. 1181928

Walkowicz, L. M., Basri, G., Batalha, N., et al. 2011, AJ, 141, 50

Waskom, M., Botvinnik, O., O'Kane, D., et al. 2018, mwaskom/seaborn: v0.9.0 (2018 July), v0.9.0, Zenodo, doi:10.5281/zenodo.1313201

West, A. A., Hawley, S. L., Bochanski, J. J., et al. 2008, AJ, 135, 785

West, A. A., Weisenburger, K. L., Irwin, J., et al. 2015, ApJ, 812, 3

Wheatley, P. J., West, R. G., Goad, M. R., et al. 2018, MNRAS, 475, 4476

Wright, N. J., Newton, E. R., Williams, P. K. G., Drake, J. J., \& Yadav, R. K. 2018, MNRAS, 479, 2351

Xu, J., Ritson, D. J., Ranjan, S., et al. 2018, Chem. Commun., 54, 5566

York, D. G., Adelman, J., Anderson, J. E. J., et al. 2000, AJ, 120, 1579

Youngblood, A., France, K., Loyd, R. O. P., et al. 2017, ApJ, 843, 31

Zhan, Z., Günther, M. N., Rappaport, S., et al. 2019, ApJ, 876, 127 